\title{
Key auxin response factor (ARF) genes constraining wheat tillering of mutant dmc
}

\author{
Junchang Li ${ }^{\text {Equal first author, } 1}$, Yumei Jiang Equal first author, ${ }^{1}$, Jing Zhang ${ }^{1}$, Yongjing Ni ${ }^{2}$, Zhixin Jiao ${ }^{1}$, Huijuan $\mathbf{L i}^{1}$, Ting Wang \\ , Peipei Zhang ${ }^{1}$, Wenlong Guo ${ }^{1}$, Lei Li ${ }^{\text {Corresp., }}{ }^{1}$, Hongjie Liu ${ }^{2}$, Hairong Zhang ${ }^{3}$, Qiaoyun Li ${ }^{1}$, Jishan Niu ${ }^{\text {Corresp. } 1}$ \\ ${ }^{1}$ National Centre of Engineering and Technological Research for Wheat/National Key Laboratory of Wheat and Maize Crop Science, Henan Agricultural \\ University, Zhengzhou, Henan, China \\ 2 Shangqiu Academy of Agricultural and Forestry Sciences, Shangqiu, Henan, China \\ 3 College of Life Sciences, Henan Agricultural University, Zhengzhou, Henan, China \\ Corresponding Authors: Lei Li, Jishan Niu \\ Email address: lilei@henau.edu.cn, jsniu@henau.edu.cn
}

Tillering ability is a key agronomy trait for wheat ( Triticum aestivum L.) production. Studies on a dwarf monoculm wheat mutant $(d m c)$ showed that ARF11 played an important role in tillering of wheat. In this study, a total of 67 ARF family members were identified and clustered to two main classes with four subgroups based on their protein structures. The promoter regions of $T$. aestivum ARF ( TaARF ) genes contain a large number of cis-acting elements closely related to plant growth and development, and hormone response. The segmental duplication events occurred commonly and played a major role in the expansion of TaARFs. The gene collinearity degrees of the ARFs between wheat and other grasses, rice and maize, were significantly high. The evolution distances among TaARFs determine their expression profiles, such as homoeologous genes have similar expression profiles, like TaARF4-3A-1, TaARF4-3A-2 and their homoeologous genes. The expression profiles of TaARFs in various tissues or organs indicated TaARF3, TaARF4, TaARF9 and TaARF22 and their homoeologous genes played basic roles during wheat development. TaARF4, TaARF9, TaARF12, TaARF15, TaARF17, TaARF21, TaARF25 and their homoeologous genes probably played basic roles in tiller development. qRT-PCR analyses of 20 representative TaARF genes revealed that the abnormal expressions of TaARF11 and TaARF14 were major causes constraining the tillering of $d m c$. Indole-3-acetic acid (IAA) contents in dmc were significantly less than that in Guomai 301 at key tillering stages. Exogenous IAA application significantly promoted wheat tillering, and affected the transcriptions of TaARFs. These data suggested that TaARFs as well as IAA signaling were involved in controlling wheat tillering. This study provided valuable clues for functional characterization of ARFs in wheat. 
1 Key auxin response factor (ARF) genes constraining wheat 2 tillering of mutant $d \boldsymbol{m c}$

3 Junchang $\mathrm{Li}^{1,}$, , Yumei Jiang ${ }^{1,}$, , Jing Zhang ${ }^{1}$, Yongjing $\mathrm{Ni}^{2}$, Zhixin Jiao ${ }^{1}$, Huijuan $\mathrm{Li}^{1}$, Ting

4 Wang ${ }^{1}$, Peipei Zhang ${ }^{1}$, Wenlong Guo ${ }^{1}$, Lei Li ${ }^{1}{ }^{1}$, Hongjie Liu ${ }^{2}$, Hairong Zhang ${ }^{3}$, Qiaoyun $\mathrm{Li}^{1}$,

5 Jishan $\mathrm{Niu}^{1, *}$

$6 \quad{ }^{1}$ Henan Agricultural University, National Centre of Engineering and Technological Research for

7 Wheat/National Key Laboratory of Wheat and Maize Crop Science, Zhengzhou, Henan, China

$8 \quad 2$ Shangqiu Academy of Agricultural and Forestry Sciences, Shangqiu, Henan, China

$9 \quad{ }^{3}$ Henan Agricultural University, College of Life Sciences, Zhengzhou, Henan, China

$10{ }^{*}$ These authors contributed equally to this work

11 Corresponding Author:

12 Jishan Niu

13 No. 15 Longzi Lake University Zone, New East District of Zhengzhou, Zhengzhou, Henan, 14 450046, China

15 Email address: jsniu@henau.edu.cn

16 Lei Li

17 No. 15 Longzi Lake University Zone, New East District of Zhengzhou, Zhengzhou, Henan, 18 450046, China

19 Email address: lilei@henau.edu.cn

20

21

22

23

24

25

26

27

28

29

30

31

32

33

34

35

36

37

38

39

\section{ABSTRACT}

Tillering ability is a key agronomy trait for wheat (Triticum aestivum L.) production. Studies on a dwarf monoculm wheat mutant $(\mathrm{dmc})$ showed that $A R F 11$ played an important role in tillering of wheat. In this study, a total of 67 ARF family members were identified and clustered to two main classes with four subgroups based on their protein structures. The promoter regions of $T$. aestivum ARF (TaARF) genes contain a large number of cis-acting elements closely related to plant growth and development, and hormone response. The segmental duplication events occurred commonly and played a major role in the expansion of TaARFs. The gene collinearity degrees of the $A R F s$ between wheat and other grasses, rice and maize, were significantly high. The evolution distances among TaARFs determine their expression profiles, such as homoeologous genes have similar expression profiles, like TaARF4-3A-1, TaARF4-3A-2 and their homoeologous genes. The expression profiles of TaARFs in various tissues or organs indicated TaARF3, TaARF4, TaARF9 and TaARF22 and their homoeologous genes played basic roles during wheat development. TaARF4, TaARF9, TaARF12, TaARF15, TaARF17, TaARF21, $T a A R F 25$ and their homoeologous genes probably played basic roles in tiller development. qRTPCR analyses of 20 representative TaARF genes revealed that the abnormal expressions of TaARF11 and TaARF14 were major causes constraining the tillering of $d m c$. Indole-3-acetic acid (IAA) contents in $d m c$ were significantly less than that in Guomai 301 at key tillering stages. Exogenous IAA application significantly promoted wheat tillering, and affected the transcriptions of TaARFs. These data suggested that TaARFs as well as IAA signaling were 
40 involved in controlling wheat tillering. This study provided valuable clues for functional 41 characterization of ARFs in wheat.

\section{Introduction}

43 Auxin response factors (ARFs) belong to a subfamily of plant B3 superfamily, and they are a 44 kind of plant-specific transcription factors (Liu et al., 2017). A large majority of ARF proteins 45 contain three conserved domains, including a N-terminal B3 DNA binding domain (DBD), a 46 middle region transcriptional activation domain (AD) or repression domain (RD), and a carboxyterminal Aux/IAA dimerization domain (CTD) (Guilfoyle and Hagen 2007; Huang et al., 2019).

As whole plant genomic sequences have been reported continuously, $A R F$ gene families in many plant species have been systematically analyzed, such as 23 ARF genes in Arabidopsis thaliana (Okushima et al., 2005), 31 ARF genes in maize (Zea mays L.) (Xing et al., 2011), 25 $A R F$ genes in rice (Oryza sativa L.) (Wang et al., 2007), 4 ARF genes in millet (Setaria italica L.) (Zhao et al., 2016), and $20 \mathrm{ARF}$ genes in barley (Hordeum vulgare L.) (Huseyin et al., 2018). These data will significantly promote the functional studies of plant $A R F$ genes.

In recent years, a large number of $A R F$ genes have been cloned in plants and some of their functions have been studied. A. thaliana ARF5 (AtARF5) is the first plant $A R F$ gene isolated by map-based cloning, and it plays an important role in the formation of embryo pattern and vascular tissue (Hardtke and Berleth 1998). Mutations in AtARF1 and AtARF2 affect the growth patterns of pistils, as well as leaf senescence, floral organ abscission (Ellis et al., 2005). AtARF3 and AtARF4 play important roles in plant reproductive and nutritional growth (Pekker et al., 2005). AtARF7 and AtARF 19 promote lateral root formation and play important roles in hormone signaling pathway (Okushima et al., 2005; Feng et al., 2012). Transgenic rice (Oryza sativa L.) lines decreasing $O$. sativa ARF1 (OSARF1) expression are low vigor, stunt growth, have short curled leaves and are sterility, which suggests that OsARF1 plays an important role in both vegetative and reproductive organ developments (Attia et al., 2009).

Tillering ability is an important agronomic trait for grain production, and tiller bud outgrowth is an important factor determining tiller number ( $\mathrm{Li}$ et al., 2003). Tiller bud growth is regulated by both genetic and environmental factors, and plant hormones are the direct regulators of both genetic and environmental factors (Zhang and Ma 2015). The endogenous hormone indole acetic acid (IAA) directly is involved in the regulation of tiller bud growth (Choi et al., 2013), IAA is mainly synthesized in the shoot tip and young leaves, and it inhibits tiller bud growth by participating in the apical dominance, thus controlling the tiller occurrence (Ljung et al., 2001).

73

74

75

76

77

78

79
ARFs regulate the expression of auxin response genes (Guilfoyle et al., 2007). Current study found that OsmiR167a repressed its targets, OsARF12, OsARF17 and OsARF25, to control rice tiller angle by fine-tuning auxin asymmetric distribution in shoots ( i et al., 2020). The transgenic rice plants overexpressing miR167 resulted in a substantial decrease the mRNA amount of four $O S A R F$ genes, OsARF6, OsARF12, OsARF17 and OsARF25, remarkably reduced tiller number (Liu et al., 2012).

At present, there are few studies on the regulation of wheat tillering by ARF genes. Research on mutant $d m c$ helped us to confirm the importance of miR396b-TaARF11 in regulating tiller 
80 development (He et al., 2018). Besides, subsequent experiments showed that the contents of IAA 81 in Guomai 301 and dmc were significantly different (An et al., 2019). In this study, all the ARF 82 family members were identified using the version of wheat reference genome (RefSeq-v1.1) 83 (IWGSC, 2018), and their evolution was studied. We thoroughly investigated the expression 84 profiles of TaARF genes in Guomai301 and mutant $d m c$ under normal growth and development 85 condition, and exogenous IAA treatment. Besides, we measured the endogenous hormone 86 contents and analyzed the correlation between IAA and tiller capacity. These results provided a 87 theoretical base for further research on the functions of ARFs in wheat.

88

89

90

91

92

93

94

95

96

97

98

99

100

101

102

103

104

105

106

107

108

109

110

111

112

113

114

115

116

117

\section{MATERIALS \& METHODS}

\section{Plant materials}

Guomai 301 is a representative semi-winter wheat cultivar in Henan, China. It has dark green leaves, thick stems, long awns, large spindle-shaped spikes, and an average 37.4 grains per spike. These data were collected as described in previous study (Li et al., 2019).

Mutant $d m c$ was obtained from EMS (ethyl methyl sulfonate) treated Guomai 301. The mutant and Guomai 301 were planted in our experimental field. Field management refer to conventional method (Li et al., 2014).

\section{Tiller sample preparation and transcriptome sequencing}

Three bulks of tiller samples were prepared separately at the three-leaf stage (WT1, dmc1; sampling date: November 15th 2018), the over-winter stage (WT2, $d m c 2$; sampling date: January 6th 2019) and the rising to jointing stage (WT3, $d m c 3$; sampling date: February 16th 2019) for RNA extraction and used for qRT-PCR analysis. Wheat tillering had been completed at the rising to jointing stage.

Tiller primordia of Guomai 301 and mutant $d m c$ at the three-leaf stage were dissected to carry out transcriptome sequencing (Fig. 1e). The tiller primordia at the three-leaf stage were carried out RNA-seq. The mutant $d m c$ (T01, T02, and T03) and WT (T04, T05, and T06) had three biological replicates, respectively. The transcript abundance of TaARFs was calculated as fragments per kilobase of exon model per million mapped reads (FPKM) (Florea et al., 2013). Differentially expressed genes (DEGs) between two sample pairs were analyzed using the DESeq R package (Wang et al., 2009). The false discovery rate (FDR $<0.01)$ and fold change (FC $\geq 2$ ) were set as the thresholds for DEGs. All analyses were performed on BMKCloud (https://www.biocloud.net/). The bioproject accession of the transcriptome data in NCBI is PRJNA670838. These data were collected as described in previous study (Li et al., 2019)

\section{Determination of endogenous hormone contents}

The tiller samples were prepared separately at the three-leaf stage (T1), the five-leaf stage (T2) and the over-winter stage (T3) for determination of endogenous hormone contents. IAA contents were extracted using a high-performance liquid chromatography method (Fang et al., 1998). Absorbance in each well was measured at $254 \mathrm{~nm}$ using a microplate reader (Thermo Scientific C18, Thermofisher, America). The samples at each stage had three independent replicates. 
118 Continuous treatment of exogenous IAA and the tiller number record

119 The IAA solution is diluted with distilled water. Data were collected as previously described 120 (Zhang et al., 2021). Specifically, the wheat seedlings of WT and mutant $d m c$ at the two-leaf 121 stage were sprayed with $10 \mu \mathrm{M}$ IAA solution on the leaves until all the leaves were wet-, and the 122 controls were sprayed with distilled water. Each seedling was sprayed with $5 \mathrm{~mL}$ of water 123 (control) or $10 \mu \mathrm{M}$ IAA solution. The samples were treated once every three days for a total of 12410 times. From the sixth time, the tiller numbers of the plants in different treatments were 125 obviously different. After then, the tiller numbers of the plants were counted every 7 days. The 126 results were analyzed using Excel for Microsoft Office 2016 according to average number.

\section{Identification and characterization of TaARFs}

128

129

130

131

132

133

134

135

136

137

138

139

140

141

142

143

144

145

146

147

148

149

150

151

152

153

154

Data were collected as previously described (Zhang et al., 2021). Specifically, the genome assembly version IWGSC refseqv1.1 (http://plants.ensembl.org/) was used to identify wheat ARF family. Considering that each gene in the wheat genome might have multiple transcripts, amino acid sequence corresponding to the longest transcript was used to identify $A R F$ gene. The prediction of ARF proteins from the wheat genome were screened using the Hidden Markov Model (HMM). The HMM files corresponding to the B3 domain (PF02362) and auxin response domain (PF06507) were downloaded from the Pfam database (http://pfam.xfam.org/). HMMER 3.3 (http://www.hmmer.org/) (Finn et al., 2011) was used to search the ARF genes from wheat genome database. All output protein sequences with e-value $\leq 1 \mathrm{e}-10$ were collected. Additionally, keywords 'ARF' and 'auxin response factor' were employed to search against the Uniprotein database (https://www.uniprot.org).

After removing all of the redundant sequences, the output putative ARF protein sequences were confirmed by CDD (https://www.ncbi.nlm.nih.gov/Structure/bwrpsb/bwrpsb.cgi), SMART (http://smart.embl-heidelberg.de/) and Pfam (http://pfam.xfam.org/) searching for the presence of the B3 domain and auxin response domain. Finally, obtained TaARFs were mainly referred to the annotation information from the Uniprotein database (https://www.uniprot.org/).

\section{Protein and gene structures, chromosomal locations of ARF genes}

The motif distribution was conducted using the MEME online tool (http://memesuite.org/tools/meme). Parameters were set as following: the motif discovery mode was classic mode, the site distribution was Zero or One Occurrence Per Sequence (zoops), the maximum number of motif finding was 8 , and other parameters were default. For exon-intron structure analysis, the DNA and cDNA sequences corresponding to each predicted protein from the wheat genome database were downloaded. The chromosomal map showed the physical location of all identified $A R F$ genes. All images were drawn using TBtools software (Chen et al., 2020). The prediction of isoelectric point $(\mathrm{pI})$ and molecular weight $(\mathrm{mw})$ of $A R F$ genes were obtained from the ExPASy Proteomics Server (https://web.expasy.org/compute_pi/).

Analysis of the cis-acting elements in TaARF promoters

Peer) reviewing PDF | (2021:06:62710:2:0:NEW 28 Aug 2021) 
155 The $2000 \mathrm{bp}$ upstream sequences of transcription start positions of TaARFs were extracted to 156 carry out the analysis of cis-acting elements. The analysis was completed using the Plant CARE 157 database (http://bioinformatics.psb.ugent.be/webtools/plantcare/html).

158 Chromosomal distribution and gene duplication

159 All $A R F$ genes were mapped to wheat chromosomes based on physical location information from 160 the database of wheat genome using Circos (Krzywinski et al., 2009). Multiple Collinearity Scan 161 toolkit (MCScanX) was adopted to analyze the gene duplication events, with the default 162 parameters (Wang et al., 2012). Non-synonymous (ka) and synonymous (ks) substitution of each 163 duplicated $A R F$ gene were calculated using KaKs Calculator 2.0 (Wang et al., 2010). The 164 syntenic maps were drawn using the Multiple Systeny Plot software (https://github.com/CJ165 Chen/TBtools)

\section{Phylogenetic analysis and classification of wheat ARF genes}

167

168

169

170

171

172

173

174

175

176

177

178

179

180

181

182

183

184

185

186

187

188

189

190

191

192

A total of $23 A R F$ genes in Arabidopsis were obtained from TAIR database (https://www.arabidopsis.org/). A total of $25 A R F$ genes in rice and $31 A R F$ genes in maize were obtained from the Uniprotein database (https:/www.uniprot.org/). The phylogenetic trees of the four species' $A R F$ genes were drawn using Neighbor-Joining (NJ) method of MEGA7.0 (http://www.megasoftware.net/), with the following parameters: Poisson model, pairwise deletion, and 1000 bootstrap replications.

\section{Analysis of ARF gene expression in various organs or tissues in wheat}

The raw gene expression data were downloaded from the Wheat Expression Browser (http://www.wheat-expression.com/). A total of 13 RNA-sequencing data from wheat cultivar Chinese Spring were analyzed. These data were prepared from 13 tissues, including seeding, root, stem, flag leaf, spike, spikelet, awn, glume, lemma, anther, grain, stamen and pistil. Gene expression levels were estimated by the transcripts per million (TPM) values, and presented as $\log _{2}$-transformed normalized TPM. The heat map was drawn by TBtools software.

\section{IAA treatment for gene expression analysis}

The seeds of Guomai 301 and $d m c$ were set in petri dishes for germination. After three days, the germinated seeds were planted in pot with soil and placed in a growth chamber at $23{ }^{\circ} \mathrm{C}$ and $50 \%$ relative humidity (RH), the light cycle was $16 \mathrm{~h}$ of light and $8 \mathrm{~h}$ of dark. The wheat seedlings at the early three-leaf stage were sprayed with distilled water, $1 \times 10^{-5} \mathrm{~mol} / \mathrm{L}$ IAA solution on the leaves, respectively. IAA was diluted with distilled water. The spray was completed until all the leaves were wet.

The tiller primordia of the seedlings sprayed with distilled water were sampled immediately and regarded as a control. The tiller primordia of the seedlings sprayed with IAA solution were sampled at $1 \mathrm{~h}$ and $2 \mathrm{~h}$ after treatments. All tiller primordia were dissected out with an anatomical needle after the out leaves and sheaths of seedlings were removed. The RNA samples of all treated tissues were immediately extracted and performed subsequent experiments.

\section{qRT-PCR}


193 Real time qRT-PCR was carried out as described in previous study (Li et al., 2019). Since the

194

195

196

197

198

199

200

201

202

203

204

205

206

207

208

209

210

211

212

213

214

215

216

217

218

219

220

221

222

223

224

225

226

227

228

229

230

homoeoalleles of most tri-genes exhibited similar expression levels (Pfeifer et al., 2014), we used universal primers to analyze the expressions of $T a A R F$ homoeoallele genes. A total of 20 pairs of primers were designed based on the consensus sequences of homoeoalleles for every wheat $A R F$ member, and the primers were listed in Table S1. The $\beta$-actin gene was used as an internal control and each reaction was performed with three biological replicates. The relative expressions of TaARFs were calculated by $2^{-\Delta \Delta C T}$ methods (Livak et al., 2001).

\section{Statistic analysis}

All data were statistically analyzed. Values shown in the form of means \pm SD were from three independent experiments. An asterisk (*) and two asterisks (**) indicate significant difference $(P<0.05)$ and highly significant difference $(P<0.01)$ using Student's $t$-tests, respectively.

\section{RESULTS}

\section{Typical traits of Guomai 301 and mutant dmc}

The mutant $d m c$ (Fig. 1a) was mutagenized from wheat cultivar Guomai 301 (Fig. 1a). Mutant $d m c$ almost didn't tiller, and only had a main stem, and the plant height of the mutant $d m c$ was significantly lower than that of the WT. At the three-leaf stage (Fig. 1b, e), two small tillers grew out at the base of the main culm in WT. Meanwhile, only one tiny protuberance formed at the main culm base of $d m c$. At the over-winter stage (Fig. 1c, f), the tiller number of WT was more than 6, while there were only two tiny tiller primordia (TPs) at the base of the $d m c$. Between the rising stage and the jointing stage (Fig. 1D), the tiny TPs of $d m c$ were almost unchanged as before (Fig. 1J); but the tiller number of WT had reached its maximum value (Fig. 1G) (An et al., 2019).

\section{The content change of endogenous IAA during wheat tiller formation}

The IAA contents in $d m c$ were significantly less than that in Guomai 301 at the three-leaf stage and the five-leaf stage (Fig. 2), and the IAA contents in Guomai 301 were 1.6-fold and 1.3-fold of that in $d m c$, respectively. While the IAA content in Guomai 301 was significantly less than that in $d m c$ at the over-winter stage, the content of IAA in $d m c$ was 5.4-fold of that in Guomai 301. Besides, the contents of IAA in Guomai 301 and $d m c$ were increased at the five-leaf stage and decreased at the over-winter stage, indicating IAA played essential roles in wheat tiller growth and development.

\section{Effects of exogenous IAA on wheat tiller formation}

On the 18th day after IAA treatment (Fig. 3, T1), the tiller number of Guomai 301 was significantly increased, while $d m c$ and the control of Guomai 301 remained no tiller. The exogenous IAA continuously promoted the tillering of Guomai 301, but the effect was less on $d m c$. (Fig. 3). The data indicated that exogenous IAA could significantly promoted tiller development of Guomai 301, but it had less effect on $d m c$, which suggested that $d m c$ was insensitive to IAA.

\section{Genome wide discovery of wheat ARFs}


231

232

233

234

235

236

237

238

239

240

241

242

243

244

245

246

247

248

249

250

251

252

253

254

255

256

257

258

259

260

261

262

263

264

265

266

267

268

A total of 74 candidate ARFs were initially obtained from all wheat protein sequences using HMM (PF02362 and PF06507) by HMMER3.3. The validation of protein conserved domains showed that 7 sequences hadn't AUX_IAA or Auxin_resp domains, which indicated that the 7 sequences were not typical ARFs. Eventually, we obtained a total of 67 unique $A R F$ genes in wheat. Detailed information about each $A R F$ gene was showed in Table S2.

Among the 67 ARF proteins, TaARF13-7D was identified as the smallest protein with 354 amino acids (aa), whereas the largest one was TaARF19-7D with 1175 aa. The molecular weight of the proteins ranged from 38829.72 $\mathrm{Da}$ (TaARF13-7D) to 130932.17 Da (TaARF19-7D), and the theoretical pI ranged from 5.42 (TaARF13-2D) to 8.7 (TaARF3-3D).

\section{Phylogenetic tree of the wheat ARF proteins}

An unrooted phylogenetic tree was generated by using the amino acid sequences of a total of 146 ARF proteins from four species (Fig. 4). The result clearly clarified the phylogenetic relationships among the ARFs. According to the bootstrap value of the phylogenetic tree, these ARFs were clustered into two classes (Class I and Class II), including four subfamilies ( I a, $\mathrm{I} \mathrm{b}, \mathbb{I} \mathrm{a}, \mathbb{I} \mathrm{b})$. Among them, the Class $I$ contained more ARF proteins.

Clustering of protein sequences from different species indicated that the ARFs in the same subfamily were highly similar, which implied their similar functions and evolution processes. Compared to Arabidopsis, wheat ARFs were more closely related to those of maize and rice.

\section{Motif pattern, domain pattern of wheat ARF proteins}

To better understand the structural characteristics of ARF proteins in each subfamily, ten conserved motifs were identified in ARF proteins using MEME motif search tool (Fig. 5b, Table S3). Only TaARF13-7D had the least number of motif modules. Motif 1, motif 3 and motif 4 modules were shared by all ARF proteins. Motif 1 , motif 2 , motif 3 , motif 4 , motif 5 , motif 6 , motif 7 , motif 9 and motif 10 modules were shared by Class $\mathbb{I}$. Typically, motif 8 existed in subfamily $\mathbb{I} \mathrm{b}$, but without in subfamily $\mathbb{I} \mathrm{a}$.

According to the result of the domain prediction, the proteins in Class I subfamily had B3 and auxin response domains. The proteins in Class II subfamily had B3, auxin response and AUX_IAA domains (Fig. 5c).

\section{Gene structure of TaARFs}

The exon-intron organizations of all the identified TaARFs were visualized (Fig. 5d). TaARFs possessed two to fourteen exons. Genes within the same group usually had similar structures. For example, all $A R F$ genes in Class II contained thirteen exons and fourteen introns, and all $A R F$ genes in Class Ia contained three exons and two introns. Among them, TaARF13-2A, TaARF132D, TaARF13-7A and TaARF13-7D had only two exons.

\section{Cis-acting elements in the promoters of TaARFs}

Among the TaARFs, the promoter sequences of 17 TaARF genes contained a large number of 'N', so they hadn't been analyzed (Fig. 6). CAT-box and CCGTCC motif cis-elements related to growth development exist commonly in the promoter sequences of TaARFs. In addition, there 
269

270

271

272

273

274

275

276

\section{7}

278

279

280

281

282

283

284

285

286

287

288

289

290

291

292

293

294

295

296

297

298

299

300

301

302

303

304

305

306

307

308

are also a large number of hormone response-related cis-elements, including some cis-acting elements involving in auxin (AuxRR-core, TGA-element), gibberellin (P-box), methyl jasmonate reaction (CGTCA-motif), salicylic acid response (TCA-element), abscisic acid response (ABRE) and ethylene response (ERE). AuxRR-core or TGA-element is the most cis-elements, 32 TaARFs contain AuxRR-core or TGA-element. Each TaARF contains at least two cis-elements. For example, TaARF25-5D has a growth-related cis-element (CAT-box) and a hormone responserelated cis-element (ABRE). These cis-acting elements implied TaARFs play various roles in regulating wheat growth and development, and respond to multiple hormones.

\section{Chromosomal localizations and duplications of TaARF genes}

The 67 TaARFs were distributed on 18 wheat chromosomes randomly. The majority of TaARFs were located on the distal ends of the chromosomes. Chromosome 7A contained the largest number of $A R F$ genes (7). No $A R F$ gene was identified on the homoeologous chromosomes 4A, 4B and 4D, and only one $A R F$ gene and its homoeologous genes (TaARF25-5A, TaARF25-5B and TaARF 25-5D) were located on homoeologous chromosomes 5A, 5B and 5D. Four pairs of tandem duplicated genes (TaARF4-3A-1 and TaARF4-3A-2, TaARF4-3B-1 and TaARF4-3B-2, TaARF4-3D-1 and TaARF4-3D-2, TaARF13-7A-1 and TaARF13-7A-2) were located on 3A, 3B, $3 \mathrm{D}$ and $7 \mathrm{~A}$, respectively. Chromosome 2D, 3A, 3B, 3D and 7A, 7B, 7D had the most $A R F$ genes.

The tandem duplication events (Fig. 7) involving chromosomal localizations of $A R F$ genes were used to directly discover the distribution of the duplication of $A R F$ genes in the wheat genome. 89 segmental duplication events among $67 A R F$ genes were identified (Table S4). In other words, all TaARFs were involved in chromosome segmental duplication. Most TaARFs were associated with two to three syntenic gene pairs. Some TaARFs had at least three syntenic gene pairs on the same chromosome, such as TaARF3-3A, TaARF3-3B, TaARF3-3D, TaARF151A, TaARF15-1B and TaARF15-1D.

Not only chromosome segmental duplication events occurred on the same chromosome, but also occurred between different chromosomes. For example, chromosome 1 and chromosome 3, chromosome 2 and chromosome 6 , a total of 19 chromosome segmental duplication events were discovered. These results indicated that the chromosome segmental duplication was a major driving force for TaARF evolution.

\section{Evolutionary relationships of ARF genes in wheat and three different species}

In order to further understand the evolution mechanism of $A R F$ genes among different species. Three comparative syntenic maps associated with wheat genome were constructed with Arabidopsis, rice and maize genomes (Fig. 8). The numbers of the orthologous $A R F$ gene pairs between wheat and the three species (Arabidopsis, rice and maize) were 6, 98 and 105, respectively (Table $\mathrm{S} 5$ ).

Six of the 67 TaARFs (TaARF22-1A, TaARF22-1B, TaARF22-1D, TaARF16-7A, TaARF16$7 B$ and TaARF16-7D) had syntenic relationship with two Arabidopsis ARF genes (AtARF10 and AtARF7) (Fig. 8a). TaARFs had higher syntenic relationship with grass plants rice and maize (Fig. 8b, c). 58 TaARFs (including 21 TaARFs and their homoeologous genes) had syntenic relationship with 19 maize $A R F$ genes (Fig. 8b), 59 TaARFs (including 21 TaARFs and their 
309 homoeologous genes) had syntenic relationship with 22 rice $A R F$ genes (Fig. 8c). Especially, the

310

311

312

313

314

315

316

317

318

319

320

321

322

323

324

325

326

327

328

329

330

331

332

333

334

335

336

337

338

339

340

341

342

343

344

345

346

347

348

syntenic gene of wheat TaARF5-6D was identified in rice, but not in maize.

The $\mathrm{Ka} / \mathrm{Ks}$ ratios of the $A R F$ gene pairs between wheat and other species (Table S5) showed that all segmental and tandem duplicated gene pairs had $\mathrm{Ka} / \mathrm{Ks}<1$, suggesting the TaARF genes might have experienced strong purifying selective pressure during evolution. In addition, the $A R F$ genes in grass plants of wheat, rice and maize were highly conserved in the syntenic blocks, for they had a closer phylogenetic relationship, and these TaARFs were evolved from ancient $A R F$ orthologous genes.

\section{The expression patterns of TaARFs in different tissues}

The expression profiles of all the 67 TaARFs during development were analyzed with the transcriptome data from the Wheat Expression Browser (http://www.wheat-expression.com/), which were derived from 13 wheat organs/tissues at different developmental stages (Fig. 9a). There were four typical expression profiles. (1) TaARFs expressed very lowly in all tissues during wheat development, such as TaARF2, TaARF8, TaARF11 and TaARF13. (2) TaARFs expressed highly in all tissues during wheat development, such as TaARF4 and TaARF9. They probably play basic important roles during wheat development. (3) TaARFs expressed in all tissues during wheat development, but the expression levels were relative lower, such as TaARF3 and TaARF22. They probably also play basic roles during wheat development. (4) TaARFs expressed highly only in specific tissues or their expression levels were changed during wheat development, such as TaARF17 expressed highly in stem and TaARF22 expressed highly in spikelet. Most TaARFs belong to this class and they play vital roles in various organ developments. Most TaARF homoeologous genes had similar expression patterns. Four pairs of tandem duplicated genes (TaARF4-3A-1 and TaARF4-3A-2, TaARF4-3B-1 and TaARF4-3B-2, TaARF4-3D-1 and TaARF4-3D-2, TaARF13-7A-1 and TaARF13-7A-2) showed remarkably different expression profiles, suggesting they evolved from different orthologous genes.

The expression profiles of TaARFS in tiller primordia showed that the transcripts of five TaARF genes (TaARF1-3A, TaARF1-3B, TaARF13-7A-2, TaARF14-1B and TaARF19-7D) had not been detected in WT and mutant $d m c$, which indicated their very lower expression levels (Fig. 9b). TaARF4, TaARF9, TaARF12, TaARF15, TaARF17, TaARF21, TaARF25 and their homoeologous genes had higher expression levels (FPKM $>10)$, but their expressions were not significant differences between WT and mutant $d m c$. High expression levels suggested they played basic important roles during tiller development. In addition, compared to WT, most TaARF genes showed low expression levels in mutant $d m c$. Only 4 TaARF genes (TaARF2-3D, TaARF11-2A, TaARF11-2B and TaARF11-2D) expressed differentially between WT and $d m c$ $(\mathrm{FC}>2)$, and they all expressed lowly in mutant $d m c$. Most TaARFs expressed relatively lower at early tillering stage in mutant $d m c$, this should be a major factor constraining tillering of the $d m c$.

In summary, TaARF3, TaARF4, TaARF9 and TaARF22 and their homoeologous genes played basic roles during wheat development. TaARF4, TaARF9, TaARF12, TaARF15, TaARF17, TaARF21, TaARF25 and their homoeologous genes probably play basic important roles during tiller development.

Peer] reviewing PDF | (2021:06:62710:2:0:NEW 28 Aug 2021) 


\section{Expression profiles of TaARFs in tiller primordia of the mutant dmc}

350 According to the transcriptomics data, most $T a A R F$ genes showed no significant differential

351

352

353

354

355

356

357

358

359

360

361

362

363

364

365

366

367

368

369

370

371

372

373

374

375

376

377

378

379

380

381

382

383

384

385

386

387

388 expressions $(\mathrm{FC}<2)$ at the three-leaf stage. qRT-PCR was performed to analyze the expression patterns of 20 TaARFs in the tiller primordia of WT and mutant $d m c$ at three tiller developmental stages (Fig. 10), and the samples at the three-leaf stage (WT1 and $d m c 1$ ) were consistent with the samples of RNA-sequencing. The $20 \mathrm{TaARF}$ genes had various expression patterns at three tillering stages.

Among them, TaARF2, TaARF3, TaARF13-2A, TaARF16 and TaARF19 showed no significant differential expressions at three tillering stages, and most $T a A R F$ genes showed no significant differential expressions at the over-winter stage, except for TaARF11 and TaARF17. At the rising to jointing stage, TaARF4, TaARF5, TaARF10, TaARF11, TaARF12, TaARF14, TaARF18 and TaARF22 had higher expression levels in mutant $d m c$. A total of $4 T a A R F$ genes showed significant differential expression levels between WT and $d m c$ at the three-leaf stage, including TaARF11, TaARF13-7A, TaARF14 and TaARF17. More importantly, these 4 TaARF genes were all down-regulated in mutant $d m c$. It indicated that only a few key genes exerted a significant effect on tiller formation at three leaf stage, the constrained tillering of the $d m c$ was associated with the lower expression levels of TaARFs. Besides, TaARF11 and TaARF14 had similar expression patterns, and they expressed lowly in $d m c$ at the over-winter stage but expressed highly at the rising to jointing stage.

In summary, the expression patterns of TaARF genes were complex. The abnormal expressions of TaARF11 and TaARF14 were major causes in constraining the tillering of $d m c$.

\section{Expression patterns of TaARFs in response to IAA}

The cis-acting element analysis showed that a number of hormone response-related cis-elements existed in the promoter regions of TaARF genes. Typically, cis-acting elements involved in auxin regulation. $20 \mathrm{TaARF}$ genes were investigated whether their expressions were affected by IAA treatment (Fig. 11).

The expressions of 6 TaARF genes (TaARF2, TaARF4, TaARF5, TaARF8, TaARF13-2A and $T a A R F 15$ ) were significantly up-regulated in mutant $d m c$ at $1 \mathrm{~h}$ after IAA treatment, among the six TaARF genes, three TaARF genes (TaARF4, TaARF5 and TaARF8) were significantly downregulated in mutant $d m c$ at $2 \mathrm{~h}$ after IAA treatment. Compare to mutant $d m c$, the expression levels of 7 TaARFs (TaARF9, TaARF11, TaARF13-2A, TaARF15, TaARF17 and TaARF21) in WT were continuously repressed by IAA treatment, especially, the expression levels of TaARF 15 and TaARF13-7A decreased by more than $50 \%$ at $1 \mathrm{~h}$ and $2 \mathrm{~h}$ after IAA treatment. TaARF13-7A had the most TGA-element (3) (Fig. 6). The promoter region of TaARF15 contained a large number of ' $\mathrm{N}$ ', so it was not analyzed. It was speculated that the auxin-related cis-acting elements determined the expressions of TaARFs response to IAA stimulating. The expressions of TaARF3 changed not significantly, which suggested it was not sensitive to IAA stimulation. Contrarily, the expressions of TaARF8 and TaARF15 were significantly affected by IAA in WT and $d m c$, which suggested they were sensitive to IAA stimulation and might play key roles in regulating wheat tillering. 
389

390

391

392

393

394

395

396

397

398

399

400

401

402

403

404

405

406

407

408

409

410

411

412

413

414

415

416

417

418

419

420

421

422

423

424

425

426

427

428

\section{Discussion}

\section{Characteristics and evolution of TaARFs}

Up to now, $A R F$ gene families have been identified in various species, including wheat. In this study, the use of multiple identification methods at the same time greatly improved the accuracy of the wheat ARF genes. A total of 23 wheat ARF members encoded by 68 homoeoalleles are identified from wheat reference genome version TGACv1 (Qiao et al., 2018), and 61 TaARF genes are identified from genome version IWGSC1+ popseq.31 (Sun et al., 2018). In this study, 67 TaARF genes, including 21 homoeologous TaARF loci, distributed on 18 chromosomes were identified in wheat using the latest version of wheat reference genome (RefSeq-v1.1) (IWGSC, 2018), which was the best version of wheat chromosome scale assembly now. The annotation of each TaARF gene was carried out referred to the Uniprotein database (https://www.uniprot.org/) Unified annotation will help these results have a wider applicability to the broader field. All these TaARFs were highly conserved, and encoded proteins with typical domains of plant ARFs.

Wheat derives from a grass ancestor structured in seven protochromosomes followed by a paleotetraploidization to reach a 12 chromosomes intermediate and a neohexaploidization (involving subgenomes A, B and D) event that finally shaped the 21 modern chromosomes (Pont et al., 2013). Because wheat is a heterohexaploid plant species, it has more $A R F$ genes than Arabidopsis (23) (Okushima et al., 2005), rice (25) (Wang et al., 2007) and maize (31) (Xing et al., 2011). The loss of $A R F$ genes on chromosome 4 (4A, 4B and 4D) might result from recombinant or modification of some redundant genes during wheat evolution (Chen et al., 2007; Otto et al., 2007). Most TaARF genes in the same subfamily have similar exon/intron structures, which provide clues to the evolutionary relationships of TaARFs (Hu et al., 2011). These data indicate that the $A R F$ genes with similar structures have similar evolution histories and functions (Babenko et al., 2004; Roy et al., 2007). A large number of cis-acting elements related to growth and development and hormones regulation existed in the promoter regions of TaARF genes, which implied their various functions. TaARFs had a poor collinearity with $A R F S$ of Arabidopsis, but had a better collinearity with $A R F S$ of rice and maize. All TaARFs might have happened segmental duplication, which had played a fundamentally important role in TaARF evolution (Zhang et al., 2000; Leister et al., 2004).

The protein sequences and gene structures of homoeologous genes TaARF4-3A-1, TaARF4$3 B-1$, and TaARF4-3D-1 were highly similar, and that of homoeologous genes TaARF4-3A-2, TaARF4-3B-2, and TaARF4-3D-2 were highly similar (Fig. 4, 5, 6), so we concluded that the two homoeologous genes evolved parallel from wheat species formation. The expression profiles of TaARF4-3A-1, TaARF4-3B-1, and TaARF4-3D-1 were similar, but were significantly different from that of TaARF4-3A-2, TaARF4-3B-2, and TaARF4-3D-2, which demonstrated the conclusion (Fig. 9). The protein and promoter sequences, and gene structures of TaARF13-7A-1 and TaARF13-7A-2 were almost the same, which indicated they were duplicated genes happened not long before (Fig. 6). Except for TaARF13-7A-1 and TaARF13-7A-2 were duplicated genes happened recently, the most TaARFs were evolved parallel from wheat species formation.

\section{Various functions of TaARFs}


429 Gene structural similarity determines its functional similarity. Plant $A R F$ genes in the same 430 subfamily have similar functions (Fig. 4). For example, disruption and overexpression of 431 AtARF8 affect hypocotyl elongation and root growth habit (Tian et al., 2004). Transgenic 432 experiments show that the $A R F 8$ can promote or inhibit lateral root formation in Arabidopsis 433 (Yang et al., 2006). AtARF4 plays an important role in the reproductive and nutritional growths 434 (Pekker et al., 2005). Similarly, TaARF4 determines root length and plant height in wheat (Wang 435 et al., 2019). These results indicated that homologous $A R F$ genes from different plant species 436 might have similar functions. Most TaARF homoeologous genes in A, B and D genomes 437 exhibited similar spatiotemporal expression profiles (Pfeifer et al., 2014), such as 438 TaARF1/4/9/12/15/17/21/25 and their homoeologous genes (Fig. 9). This data also suggested the 439 homoeologous TaARFs had similar functions.

441

442

443

444

445

446

447

448

449

450

451

452

453

454

455

456

457

458

459

460

461

462

463

464

465

466

467

468

Most $A R F$ genes have different tissue-specific expression patterns, suggesting their special functions in different tissue/organ development. For example, $A R F 7$ and $A R F 19$ regulate lateral root formation in Arabidopsis (Fukaki et al., 2006; Okushima et al., 2007). Transgenic Arabidopsis lines expressing TaARF15-A.1 promotes the growth of roots and leaves (Qiao et al., 2018). OsARF19 is pivotal for floral organ development and plant architecture (Zhang et al., 2015). ARF17 is essential for pollen wall patterning in Arabidopsis by modulating primexine formation at least partially through direct regulation of CalS5 gene expression (Yang et al., 2013), and the overexpression of ARF17 in the tapetum and microsporocytes of $5 m A R F 17 / \mathrm{WT}$ plants leads to male sterility (Wang et al., 2017). Overexpression of AtTTP affects ARF17 expression and leads to male sterility in Arabidopsis (Shi et al., 2015). Up to now, most functional studies of $A R F$ genes have been carried out in A. thaliana. Most TaARFs also have typical tissue-specific expression profiles (Fig. 9), which suggests their various functions in wheat development.

\section{The key TaARFs involved in tiller development}

Plant $A R F$ genes play an important role in maintaining plant stem apical meristem (Zhao et al., 2010). The enhanced miR167 level in transgenic rice resulted in a substantial decrease in mRNA amounts of the four OsARF genes, OsARF6, OsARF12, OsARF17 and OsARF25, the transgenic rice plants remarkably reduced tiller number (Liu et al., 2012). Recent research suggested OsmiR167a could repress $O S A R F 12, O S A R F 17$ and $O S A R F 25$, to control rice tiller angle by finetuning auxin asymmetric distribution in shoots ( $\mathrm{Li}$ et al., 2020). Our miRNome and transcriptome integrative analysis about the mutant $d m c$ and WT found that the highly expressed tae-miR396b ( $T$. aestivum microRNA396b) significantly repressed the expressions of TaGRF genes and TaARF11 in $d m c$ during tillering (He et al., 2018). It was predicted that the miR396b/ARF11 regulatory module played a key role in wheat tiller development. Compared with the WT, the expressions of four TaARFs, TaARF11, TaARF13-7A, TaARF14 and TaARF17, in $d m c$ were significantly decreased at early tillering stage, which was positively related to the phenotype of $d m \mathrm{c}$ (Fig. 10). Most TaARFs had different expression patterns in WT and $d m c$, but only those significantly differentially expressed TaARFs in tiller primordia were the key tiller development regulators. In this case, TaARF11 and TaARF14 were significantly differentially 
469 expressed at early tillering stage, indicating their important roles in regulating tiller numbers in 470 wheat.

\section{IAA affect the expressions of TaARFs and significantly promoted tillering}

472 Hormone responses are fundamental to the development and plastic growth of plants (Chapman 473 et al., 2009). There are a number of evidences that exogenous IAA can obviously influence rice 474 and wheat tillering (Kariali et al., 2007; Liu et al., 2011; Assuero et al., 2012; Cai et al., 2013). 475 Apically derived auxin does not enter axillary buds directly in several species, including in 476 Arabidopsis (Booker et al., 2003). Apical auxin can inhibit the growth of small buds, and it has 477 been proposed that its inhibitory effect is mediated by a second messenger (Chatfield et al., 478 2001). In rice, there are many genes related to tiller number may also be related to various plant 479 hormones, rice dwarf and low tillering 10 (OsDLT10) regulates tiller number by monitoring 480 auxin homeostasis (Wen et al., 2020). The phytohormone auxin is involved in almost all 481 developmental processes in land plants, different $A R F$ genes probably contribute to the 482 establishment of multiple unique auxin responses in plant development (Roosjen et al., 2017). In 483 our study, the TaARF genes showed various expression patterns after IAA treatment. There are a 484 large number of cis-acting elements related to hormones in TaARF promoters, including those 485 related to IAA (AuxRR-core, TGA-element). Tissue specific promoters control gene expression 486 in certain organs or tissues ( $\mathrm{Li}$ et al., 2015). The results of qRT-PCR also confirmed that the 487 expressions of TaARFs were significantly affected by IAA treatment (Fig. 11). IAA contents in $488 d m c$ were significantly less than that in Guomai 301 at key tillering stages (Fig. 2), and IAA 489 application significantly promoted wheat tillering (Fig. 3). According to these data, it was 490 considered that TaARFs as well as IAA signaling were involved in regulating wheat tiller 491 development.

492

493

494

495

496

497

498

499

500

501

502

503

504

505

506

\section{Conclusions}

A total of 67 TaARFs were identified in wheat. TaARF genes distribute on 18 wheat chromosomes randomly, and their promoter regions have a large number of cis-acting elements related to plant growth and development, and hormone response. The most TaARFs evolved parallel from wheat formation, except for TaARF13-7A-1 and TaARF13-7A-2 duplicated recently. The homoeologous TaARFs are highly similar and also have similar expression profiles. TaARF3, TaARF4, TaARF9 and TaARF22 and their homoeologous genes play basic roles during wheat development. TaARF4, TaARF9, TaARF12, TaARF15, TaARF17, TaARF21, TaARF25 and their homoeologous genes play basic roles during tiller development. The abnormal expressions of TaARF11 and TaARF14 are major causes constraining the tillering of $d m c$. The IAA contents of $d m c$ are significantly less than that in WT during key tillering stages. Exogenous IAA significantly affected the expressions of TaARFs and promoted wheat tillering, which demonstrated that TaARFs and IAA signaling were involved in controlling wheat tillering. This study provided valuable clues for functional characterization of ARFs in wheat.

\section{Acknowledgements}


507 We are grateful for the assistance by Shangqiu Academy of Agricultural and Forestry Sciences.

508 We thank National Centre of Engineering and Technological Research of Wheat for the technical

509 support for the cultivations.

510 References

511 An J, Niu H, Ni Y, Jiang Y, Zheng Y, He R, Li J, Jiao Z, Zhang J, Li H, Li Q, Niu J. 2019.

512 The miRNA-mRNA networks involving abnormal energy and hormone metabolisms

513 restrict tillering in a wheat mutant dmc. International Journal of Molecular Sciences 20:

514

515 4586. DOI: 10.3390/ijms20184586

Assuero SG, Lorenzo M, Ramírez NMP, Velázquez LM, Tognetti JA. 2012. Tillering

516 promotion by paclobutrazol in wheat and its relationship with plant carbohydrate status.

517 New Zealand Journal of Agricultural Research 55: 347-358. DOI:

518

519 10.1080/00288233.2012.706223

Attia KA, Abdelkhalik AF, Ammar MH, Wei C, Yang J, Lightfoot DA, EI-Sayed WM, EIShemy HA. 2019. Antisense phenotypes reveal a functional expression of OsARF1, an auxin response factor, in transgenic rice. Current Issues in Molecular Biology 11: 29-34.

521

522

523 DOI: $10.21775 / 9781912530069.04$

Babenko V N, Rogozin I B, Mekhedov S L, Koonin E V. 2004. Prevalence of intron gain over

524 intron loss in the evolution of paralogous gene families. Nucleic Acids Research 32: 3724-

525

526

527 3733. DOI: 10.1093/nar/gkh686

Booker J, Chatfield S, Leyser O. 2003. Auxin acts in xylem-associated or medullary cells to mediate apical dominance. Plant Cell, 15: 495-507. DOI: 10.1105/tpc.007542

528

Cai T, Xu HC, Yin YP, Yang WB, Wang ZL. 2013. Mechanisms of tiller occurrence affected

529 by exogenous IAA, GA, and ABA in wheat with different spike-types. Acta Agronomica

530 Sinica 39: 1835. DOI: 10.3724/SP.J.1006.2013.01835

531

532

Chapman EJ, Estelle M. 2009. Mechanism of auxin-regulated gene expression in plants.

533 Annual Review of Genetics 43: 265-285. DOI: 10.1146/annurev-genet-102108-134148

534

535

Chatfield S P, Stirnberg P, Forde B G, Leyser O. 2000. The hormonal regulation of axillary bud growth in Arabidopsis. Plant Journal 24: 159-169. DOI: 10.1046/j.1365313x.2000.00862.x

536

Chen C, Chen H, Zhang Y, Thomas HR, Frank MH, Hem Y, Xia R. 2020. TBtools - an integrative toolkit developed for interactive analyses of big biological data. Molecular Plant

538 13: 1194-1202. DOI: 10.1016/j.molp.2020.06.009

539 Chen ZJ. 2007. Genetic and epigenetic mechanisms for gene expression and phenotypic

540 variation in plant polyploids. Annual Review of Plant Biology 58: 377-406. DOI:

541 10.1146/annurev.arplant.58.032806.103835

542

543

Choi M, Koh E, Woo M, Piao R, Oh C, Koh H. 2013. Tiller formation in rice is altered by overexpression of $O S I A G L U$ gene encoding an IAA-conjugating enzyme or exogenous

544

545 treatment of free IAA. Journal of Plant Biology 55: 429-435. DOI: 10.1007/s12374-012-02380 
546 Ellis CM, Nagpal P, Young JC, Hagen G, Guilfoyle TJ, Reed JW. 2005. AUXIN RESPONSE FACTOR1 and AUXIN RESPONSE FACTOR2 regulate senescence and floral organ abscission in Arabidopsis thaliana. Development 132: 4563-4574. DOI:

550

551

552

553

554

555

556

557

558

559

560

561

562

563

564

565

566

567

568

569

570

571

572

573

574

575

576

577

578

579

580

581

582

583

584 $10.1242 / \mathrm{dev} .02012$

Fang N, Hou S, Shao X, He Y, and Zhao G. 1998. Separation and determination of eight plant hormones by reversed phase high performance liquid chromatography. Chinese Journal of Chromatography 16: 417. DOI: 10.1088/0256-307X/16/12/013

Feng Z, Zhu J, Du X, Cui X. 2012. Effects of three auxin-inducible LBD members on lateral root formation in Arabidopsis thaliana. Planta 236(4): 1227-1237. DOI: 10.1007/s00425-0121673-3

Finn RD, Clements J, Eddy SR. 2011. HMMER web server: interactive sequence similarity searching. Nucleic Acids Research 39: W29-W37. DOI: 10.1093/nar/gkr367

Florea L, Song L, Salzberg SL. 2013. Thousands of exon skipping events differentiate among splicing patterns in sixteen human tissues. F1000Research 2: 188 . DOI: 10.12688/f1000research.2-188.v1

Fukaki H, Taniguchi N, Tasaka M. 2006. PICKLE is required for SOLITARY-ROOT/IAA14mediated repression of ARF7 and ARF19 activity during Arabidopsis lateral root initiation. The Plant Journal 48: 380-389. DOI: 10.1111/j.1365-313X.2006.02882.x

Guilfoyle TJ, Hagen G. 2007. Auxin response factors. Current Opinion Plant Biology 10: 453460. DOI: 10.1016/j.pbi.2007.08.014

Hardtke CS, Berleth T. 1998. The Arabidopsis gene MONOPTEROS encodes a transcription factor mediating embryo axis formation and vascular development. The EMBO Journal 17: 1405-1411. DOI: 10.1093/emboj/17.5.1405

He R, Ni Y, Li J, Jiao Z, Zhu X, Jiang Y, Li Q, Niu J. 2018. Quantitative changes in the transcription of phytohormone-related genes: some transcription factors are major causes of the wheat mutant $d m c$ not tillering. International Journal of Molecular Sciences 19: 1324. DOI: 10.3390/ijms 19051324

Hu L, Liu S. 2011. Genome-wide identification and phylogenetic analysis of the ERF gene family in cucumbers. Genetics and Molecular Biology 34: 624-634. DOI: 10.1590/s141547572011005000054

Huang X, Zhang T, Liu C, Zhao Y, Wei H, Zhou J, Yuan Z. 2019. Genome-wide identification and expression analysis of auxin response factor (ARF) gene family in Punica granatum. Journal of Fruit Science 36: 43-55. DOI: 10.13925/j.cnki.gsxb.20180164

Huseyin, T. 2018. Genome-wide analysis of the auxin response factors (ARF) gene family in barley (Hordeum vulgare L.). Journal of Plant Biochemistry and Biotechnology 28: 14-24. DOI: $10.1007 / \mathrm{s} 13562-018-0458-6$

International Wheat Genome Sequencing Consortium (IWGSC). 2018. Shifting the limits in wheat research and breeding using a fully annotated reference genome. Science 361: 661. DOI: 10.1126/science.aar7191 
585 Kariali E, Mohapatra PK. 2007. Hormonal regulation of tiller dynamics in differentially-

586

587

588

589

590

591

592

593

594

595

596

597

598

599

600

601

602

603

604

605

606

607

608

609

610

611

612

613

614

615

616

617

618

619

620

621

622

623

tillering rice cultivars. Plant Growth Regulation 53: 215-223. DOI: 10.1007/s10725-007-9221Z

Krzywinski M, Schein J, Birol I, Connors J, Gascoyne R, Horsman D, Jones SJ, Marra MA. 2009. Circos: An information aesthetic for comparative genomics. Genome Research 19: 1639-1645. DOI: 10.1101/gr.092759.109

Leister D. 2004. Tandem and segmental gene duplication and recombination in the evolution of plant disease resistance genes. Trends in Genetics 20: 116-122. DOI: 10.1016/j.tig.2004.01.007

Li J, Jiao Z, He R, Sun Y, Xu Q, Zhang J, Jiang Y, Li Q, Niu J. 2019. Gene expression profiles and microRNA regulation networks in tiller primordia, stem tips, and young spikes of wheat Guomai 301. Genes 10: 686. DOI: 10.3390/genes10090686

Li Q, Qin Z, Jian, Y, Shen C, Duan Z, Niu J. 2014. Screening wheat genotypes for resistance to black point and the effects of diseased kernels on seed germination. Journal of Plant Diseases and Protection 121: 79-88. DOI: 10.1007/BF03356495

Li X, Qian Q, Fu Z, Wang Y, Xiong G, Zeng D, Wang X, Liu X, Teng S, Hiroshi F, Yuan M, Luo D, Han B, Li J. 2003. Control of tillering in rice. Nature 422: 618. DOI: 10.1038/nature 01518

Li Y, Li J, Chen Z, Wei Y, Qi Y, Wu C. 2020. OsmiR167a-targeted auxin response factors modulate tiller angle via fine-tuning auxin distribution in rice. Plant Biotechnol Journal pp. 112. DOI: $10.1111 / \mathrm{pbi} .13360$

Li Z, Chen X. 2015. Research advances in plant tissue specific promoters and related Cis-acting elements. Journal of Biology 32: 91-95. DOI: 10.3969/j.issn.2095-1736.2015.06.091

Liu H, Jia S, Shen D, Liu j, Li J, Zhao H, Han S, Wang Y. 2012. Four AUXIN RESPONSE FACTOR genes downregulated by microRNA167 are associated with growth and development in Oryza sativa. Functional Plant Biology 39: 736-744. DOI: 10.1071/FP12106

Liu, Y, Dong Z. 2017. The function and structure of plant B3 domain transcription factor. Molecular Plant Breeding 15: 1868-1873. DOI: 10.13271/j.mpb.015.001868

Liu Y, Gu D, Ding Y, Wang Q, Li G, Wang S. 2011. The relationship between nitrogen, auxin and cytokinin in the growth regulation of rice (Oryza sativa L.) tiller buds. Australian Journal of Crop Science 5: 1019. DOI: 10.1111/j.1439-0523.2010.01842.x

Livak KJ, Schmittgen TD. 2001. Analysis of relative gene expression data using realtime quantitative PCR and the 2- $\triangle \mathrm{CCT}$ method. Methods 25: 402-408. DOI: 10.1006/meth.2001.1262

Ljung K, Bhalerao RP, Sandberg G. 2001. Sites and homeostatic control of auxin biosynthesis in Arabidopsis during vegetative growth. The Plant Journal 28: 465-474. DOI: 10.1046/j.1365-313X.2001.01173.X

Okushima Y, Fukaki H, Onoda M, Theologis A, Tasaka M. 2007. $A R F 7$ and $A R F 19$ regulate lateral root formation via direct activation of LBD/ASL genes in Arabidopsis. Plant Cell 19:118-130. DOI: $10.1105 /$ tpc.106.047761

Peer) reviewing PDF | (2021:06:62710:2:0:NEW 28 Aug 2021) 
624 Okushima Y, Overvoordea PJ, Arima K, Alonso JM, Chan A, Chang C, Ecker JR, Hughes

625

626

627

628

629

630

631

632

633

634

635

636

637

638

639

640

641

642

643

644

645

646

647

648

649

650

651

652

653

654

655

656

657

658

659

660

661

662

B, Lui A, Nguyen D, Onodera C, Quach H, Smith A, Yu G, Theologis A. 2015. Functional genomic analysis of the AUXIN RESPONSE FACTOR gene family members in Arabidopsis thaliana: unique and overlapping functions of ARF7 and ARF19. The Plant Cell 17: 444-463. DOI: $10.1105 /$ tpc.104.028316

Otto SP. 2007. The evolutionary consequences of polyploidy. Cell 131: 452-462. DOI: 10.1016/j.cell.2007.10.022

Pekker I, Alvarez J.P, Eshed Y. 2005. Auxin response factors mediate Arabidopsis organ asymmetry via modulation of KANADI activity. The Plant Cell 17: 2899-2910. DOI: 10.1105/tpc.105.03

Pfeifer M, Kugler KG, Sandve SR, Zhan B, Rudi H, Hvidsten TR, IWGSC, Mayer KFX, Olsen O. 2014. Genome interplay in the grain transcriptome of hexaploid bread wheat. Science 345: 1250091. DOI: $10.1126 /$ science. 1250091

Pont C, Murat F, Guizard S, Flores R, Foucrier S, Bidet Y, Quraishi U, Alaux M, Dolezel J, Fahima T, Budak H, Keller B, Salvi S, Maccaferri M, Steinbach D, Feuillet C, Quesneville H. 2013. Wheat syntenome unveils new evidences of contrasted evolutionary plasticity between paleo- and neoduplicated subgenomes. The Plant Journal 76: 1030-1044. DOI: $10.1111 /$ tpj.12366

Qiao L, Zhang W, Li X, Zhang L, Zhang X, Li X, Guo H, Ren Y, Zheng J, Chang Z. 2018. Characterization and expression patterns of auxin response factors in wheat. Frontiers in Plant Science 9: 1395. DOI: 10.3389/fpls.2018.01395

Roosjen M, Paque S, Weijers D. 2017. Auxin response factors: output control in auxin biology. Journal of Experimental Botany 69: 179-188. DOI: 10.1093/jxb/erx237

Roy SW, Penny D. 2007. On the incidence of intron loss and gain in paralogous gene families. Molecular Biology and Evolution 24: 1579-1581. DOI: 10.1093/molbev/msm082

Shi ZH, Zhang C, Xu X, Zhu J, Zhou Q, Ma L, Niu J, Yang Z. 2015. Overexpression of AtTTP affects arf17 expression and leads to male sterility in Arabidopsis. PLoS One 10: e0117317. DOI: 10.1371/journal.pone.0117317

Sun R, Liu Y, Wang X, Zhang Q, Zhang L, Sun H, Wang Y, Gao J, Yang W, Zhao C, Gao S, Han J. 2018. Bioinformatics identification of auxin response factor genes and expression profiles under drought stress in wheat. Journal of Plant Genetic Resources 19: 122-134. DOI: 10.13430/j.cnki.jpgr.2018.01.014

Tian CT, Muto H, Higuchi K, Matamura T, Tatematsu K, Koshiba T, Yamamoto KT. 2004. Disruption and overexpression of auxin response factor 8 gene of Arabidopsis affect hypocotyl elongation and root growth habit, indicating its possible involvement in auxin homeostasis in light condition. The Plant Journal 40: 333-343. DOI: 10.1111/j.1365-313X.2004.02220.x

Wang B, Xue J, Yu Y, Liu S, Zhang J, Yao X, Liu Z, Xu X, Yang Z. 2017. Fine regulation of ARF17 for anther development and pollen formation. BMC Plant Biology 17: 243. DOI: 10.1186/s12870-017-1185-1

Peer] reviewing PDF | (2021:06:62710:2:0:NEW 28 Aug 2021) 
663 Wang D, Pei K, Fu Y, Sun Z, Li S, Liu H, Tang K, Han B, Tao Y. 2007. Genome-wide analysis of the auxin response factors. ARF. gene family in rice. Oryza sativa. Gene 394: 1324. DOI: 10.1016/j.gene.2007.01.006

666

Wang D, Zhang Y, Zhang Z, Zhu J, Yu J. 2010. KaKs_Calculator 2.0: a toolkit incorporating 667 gamma-series methods and sliding window strategies. Genomics Proteomics Bioinformatics 8:

668 77-80. DOI: 10.1016/S1672-0229(10)60008-3

669

Wang J, Wang R, Mao X, Li L, Chang X, Zhang X, Jing R. 2019. TaARF4 genes are linked 670 to root growth and plant height in wheat. Annals of Botany 124: 903-915. DOI:

671 10.1093/aob/mcy218

672

Wang L, Feng Z, Wang X, Wang $X$, Zhang X. 2009. DEGseq: an R package for identifying

673 differentially expressed genes from RNA-seq data. Bioinformatics 26:136-148. DOI:

674 10.1093/bioinformatics/btp612

675

676

Wang Y, Tang H, DeBarry JD, Tan X, Li J, Wang X, Lee T, Jin H, Marler B, Guo H, Kissinger JC, Paterson AH. 2012. MCScanX: a toolkit for detection and evolutionary

677 analysis of gene synteny and collinearity. Nucleic Acids Research 40: e49. DOI:

678 10.1093/nar/gkr1293

679

Wen X, Sun, Chen Y, Xue P, Yang Q, Wang B, Yu N, Cao Y, Zhang Y, Gong K, Wu W,

680

681 Chen D, Cao L, Cheng S, Zhang Y, Zhan X. 2020. Rice dwarf and low tillering 10

682 (OsDLT10) regulates tiller number by monitoring auxin homeostasis. Plant Science 297:

683 110502. DOI: 10.1016/j.plantsci.2020.110502

684

Xing H, Pudake RN, Guo G, Xing G, Hu Z, Zhang Y, Sun Q, Ni Z. 2011. Genome-wide identification and expression profiling of auxin response factor. ARF. gene family in maize.

685 BMC Genomics. 12: 178. DOI: 10.1186/1471-2164-12-178

686

687

Yang J, Tian L, Sun M, Huang X, Zhu J, Guan Y, Jia Q, Yang Z. 2013. AUXIN

688 RESPONSE FACTOR17 is essential for pollen wall pattern formation in Arabidopsis. Plant Physiology 162: 720-731. DOI: 10.1104/pp.113.214940

689

Yang JH, Han SJ, Yoon EK, Lee WS. 2006. Evidence of an auxin signal pathway, microRNA167-ARF8-GH3, and its response to exogenous auxin in cultured rice cells. Nucleic

691 Acids Research 34: 1892-1899. DOI: 10.1093/nar/gk1118

692

Zhang J, Li J, Ni Y, Jiang Y, Jiao Z, Li H, Wang T, Zhang P, Han M, Li L, Liu H, Li Q, Niu J. 2021. Key wheat GRF genes constraining wheat tillering of mutant dmc. PeerJ 9:

694 e11235. DOI 10.7717/peerj.11235

695

Zhang PA. 2000. Segmental gene duplication generated differentially expressed MYBhomologous genes in maize. The Plant Cell 12: 2311-2322. DOI: 10.1105/tpc.12.12.2311

697

Zhang S, Ma H. 2015. Hormone regulation and tillering ability of different kentucky bluegrass

698 varieties. Acta Agrestia Sinica 23: 316-321. DOI: 10.11733/j.issn.1007-0435.2015.02.015

699

Zhang S, Wu T, Liu S, Liu X, Jiang L, Wan J. 2015. Disruption of OsARF19 is critical for 700 floral organ development and plant architecture in rice (Oryza sativa L.). Plant Molecular 701 Biology Reporter 34: 748-760. DOI: 10.1007/s11105-015-0962-y 
702 Zhao Y, Weng Q, Ma H, Song J, Yuan J, Wang L, Dong Z, Liu Y. 2016. Genome-wide 703 identification and bioinformatics analysis of ARF gene family in foxtail millet Setaria italic.

704 Journal of Plant Genetic Resources 17: 547-554. DOI: 10.13430/j.cnki.jpgr.2016.03.022

705 Zhao Z, Andersen SU, Ljung K, Dolezal K, Miotk A, Schultheiss SJ, Lohmann JU. 2010.

706 Hormonal control of the shoot stem-cell niche. Nature 465. DOI: 10.1038/nature09126 


\section{Figure 1}

The tiller micromorphology of Guomai 301 (left) and mutantdmc(right).

(a), The individual plants of Guomai 301 and mutantdmcin the field condition. (b), The seedlings of Guomai 301anddmcat the three-leaf stage. (c), The seedlings of Guomai 301 anddmcat the over-winter stage; (d), The seedlings of Guomai 301 anddmcat the rising to jointing stage. (e), Tiller primordia of Guomai 301 anddmcat the three-leaf stage. (f), Tiller primordia of Guomai 301 anddmcat the over-winter stage. (g), Tiller primordia of Guomai 301 anddmcat the rising to jointing stage. MC: main culm; TP: tiller primordium; Scale bar: $10 \mathrm{~cm}$ (a); $2 \mathrm{~cm}(\mathrm{~b}-\mathrm{d}) ; 1 \mathrm{~cm}(\mathrm{e}-\mathrm{g})$.

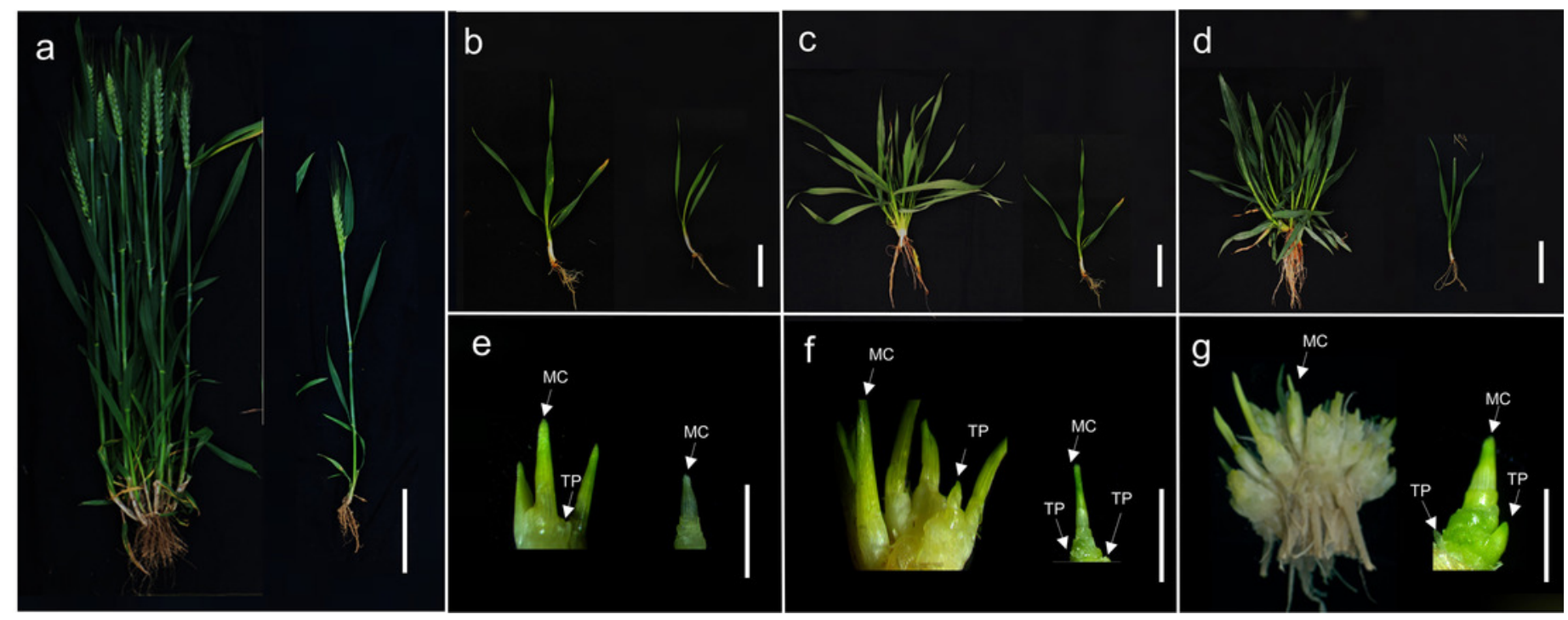


Figure 2

The endogenous IAA contents in tiller primordia of Guomai301 and $d m c$.

S1: the three-leaf stage; S2: the five-leaf stage; S3: the over-winter stage. Asterisks indicate significant difference or highly significant difference between Guomai 301 and dmc in different stages.

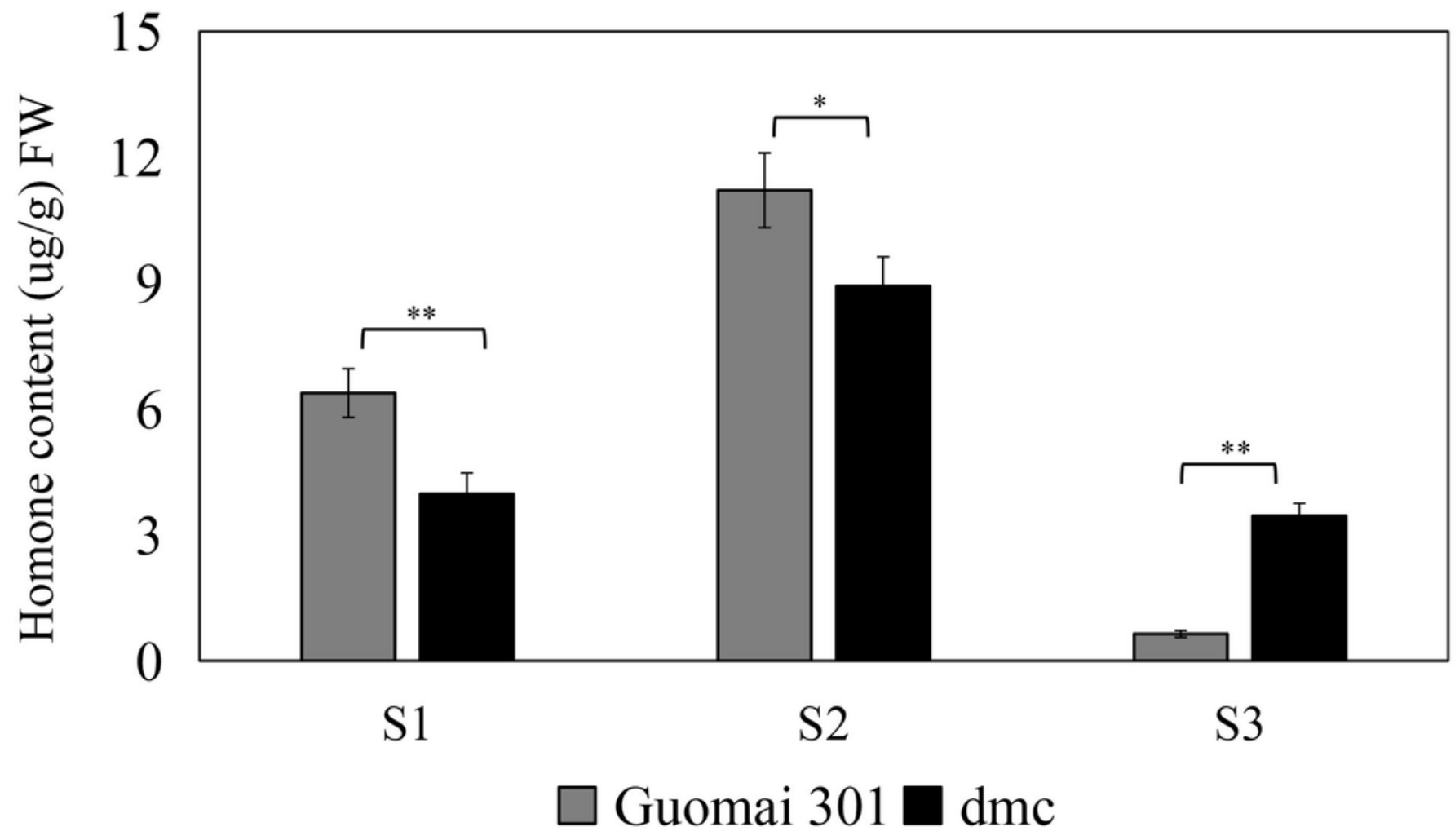


Figure 3

The tiller number changes of Guomai 301 and $d m c$ in response to IAA treatments.

T1-T6 of the $x$-axis indicated the sampling dates, and the tiller numbers were recorded every 7 days. $T 1$ is the first sampling date which was the 18th day after IAA treatment. Asterisks indicate significant difference or highly significant difference between treated groups and control groups in different sampling dates, respectively.

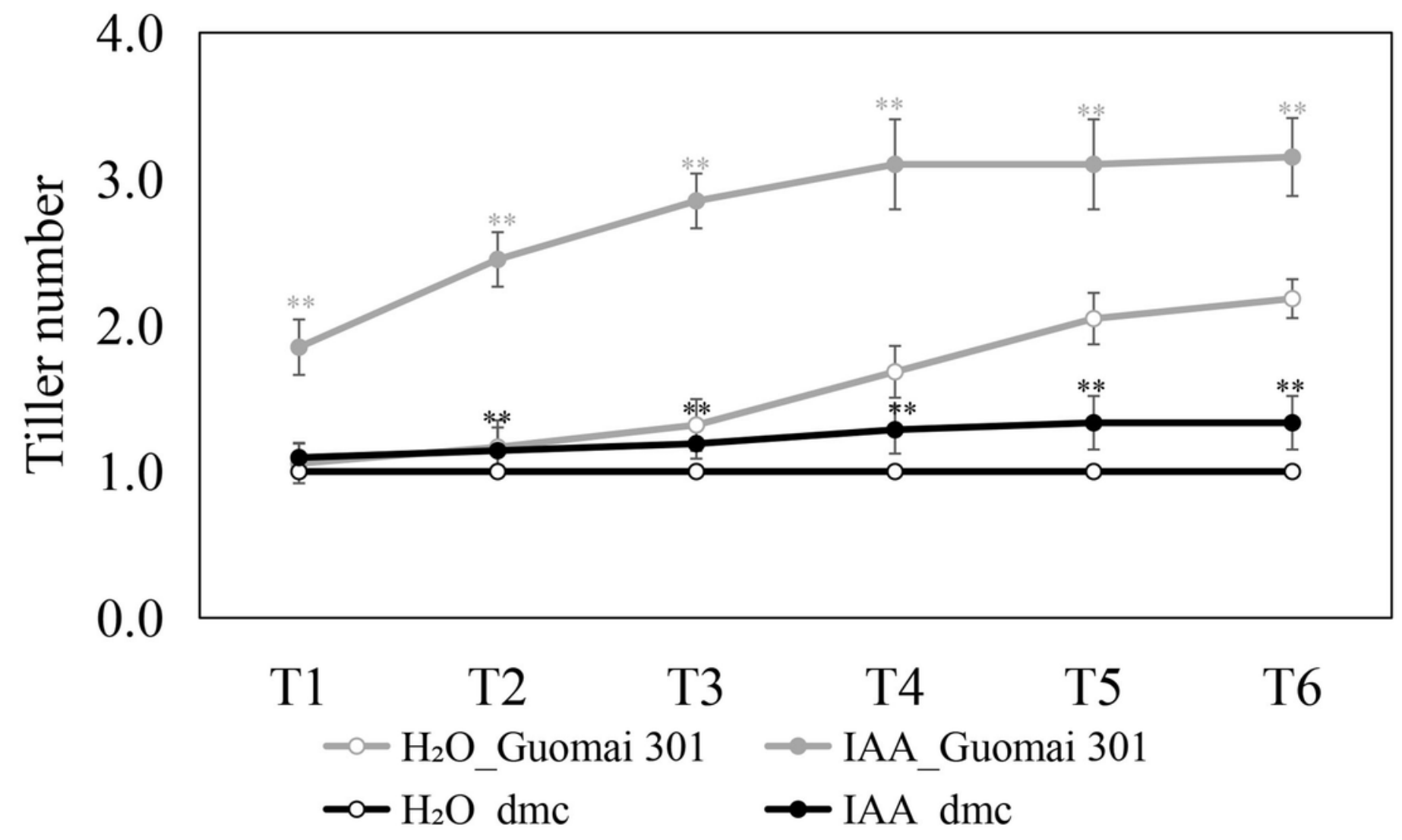




\section{Figure 4}

Phylogenetic tree of ARF proteins from Arabidopsis, maize, rice and wheat.

The purple solid diamonds represent ARF proteins in Arabidopsis (AtARF); The green squares represent ARF proteins in maize (ZmARF); The blue deltas represent ARF proteins in rice (OsARF); The red solid circles represent ARF proteins in wheat (TaARF); The different colored sectors indicate different groups (or subgroups) of ARF proteins. The different colored arcs indicate different classes of ARF proteins. 


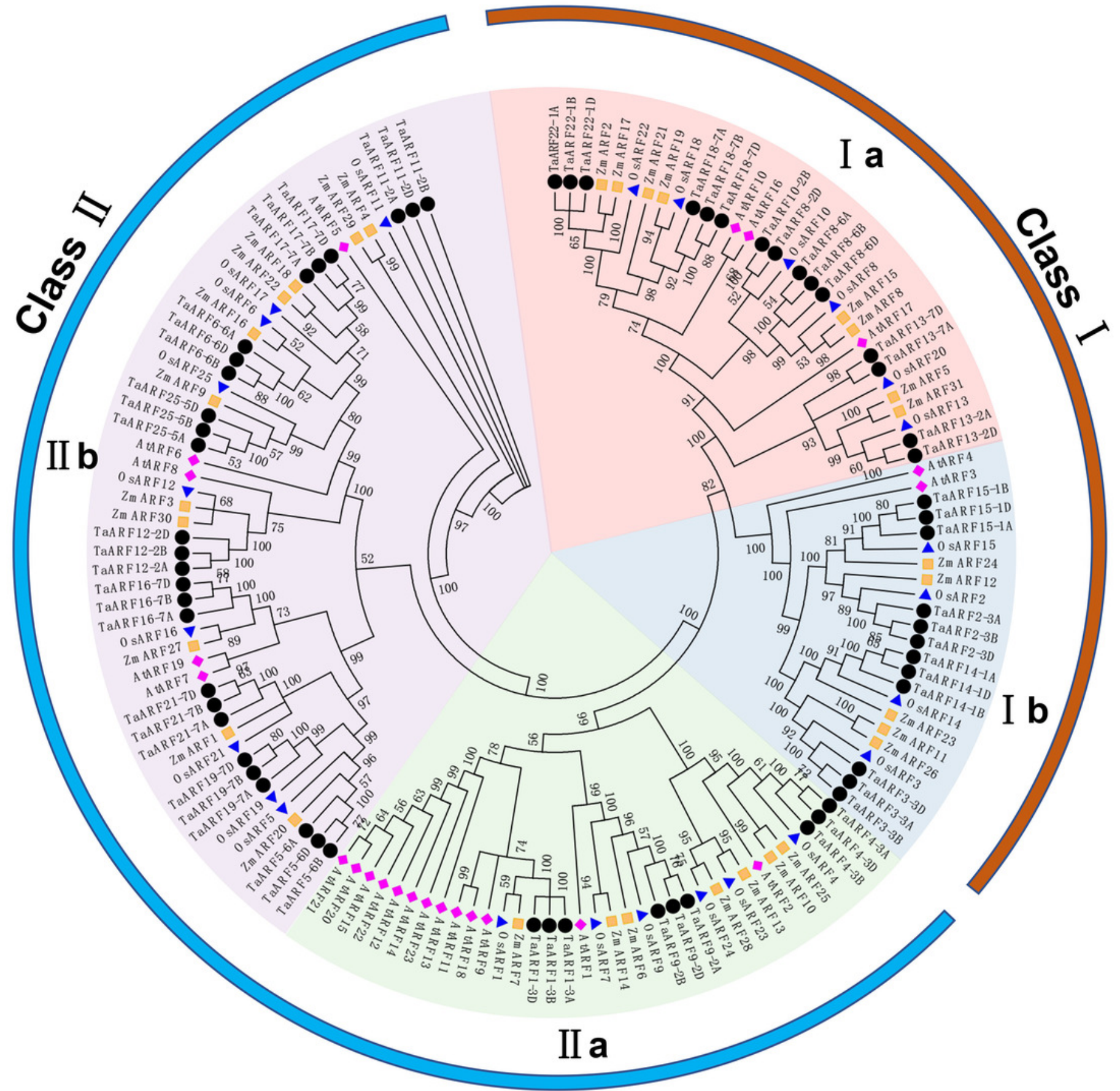




\section{Figure 5}

Phylogenetic relationships, conserved protein motif patterns, domain patterns and gene structures of TaARFs.

(a), the phylogenetic tree of TaARF proteins. Clusters are indicated with different colors. (b), The motif compositions of TaARFs. The 1-10 motifs are displayed in different colored boxes, the scale at the bottom indicates the length of proteins. (c), The domain patterns of TaARFs, the $B 3$ domains are highlighted in yellow, the auxin response domains are highlighted in green, and the AUX_IAA domain are highlighted in lilac. (d), exon-intron structures of TaARFs, yellow boxes indicate $5^{\prime}$ - and 3 '- untranslated regions; green boxes indicate exons; black lines indicate introns. 


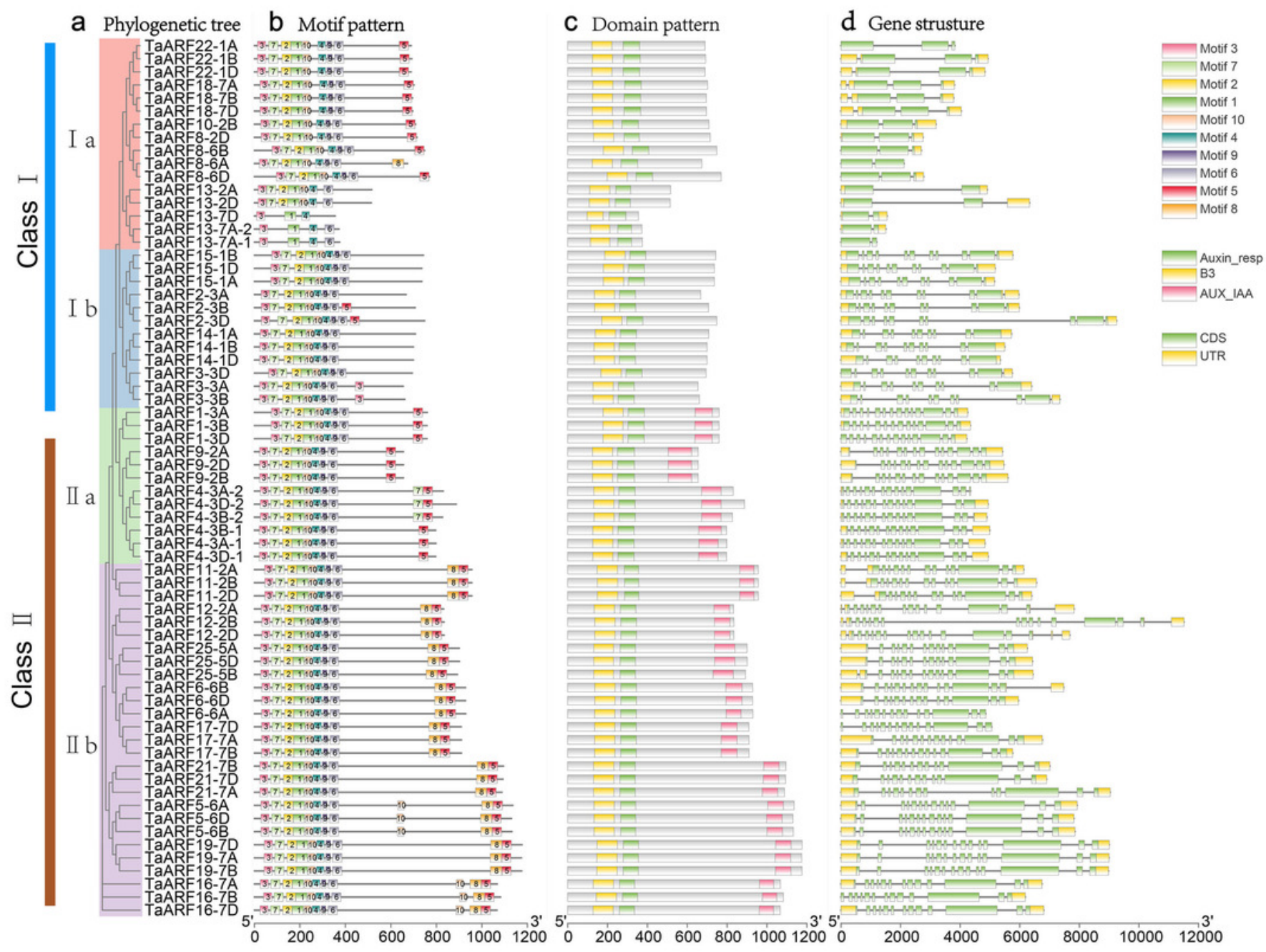




\section{Figure 6}

The cis-acting elements in the promoters of TaARFs.

Growth-related cis-element: meristem expression regulation (CAT-box and CCGTCC motifs); hormone response-related cis-elements: abscisic acid response (ABRE), methyl jasmonate response (CGTCA-motif), salicylic acid response (TCA-element), gibberellic response (P-box), auxin response (TGA-element and AuxRR-core) and ethylene response (ERE). 


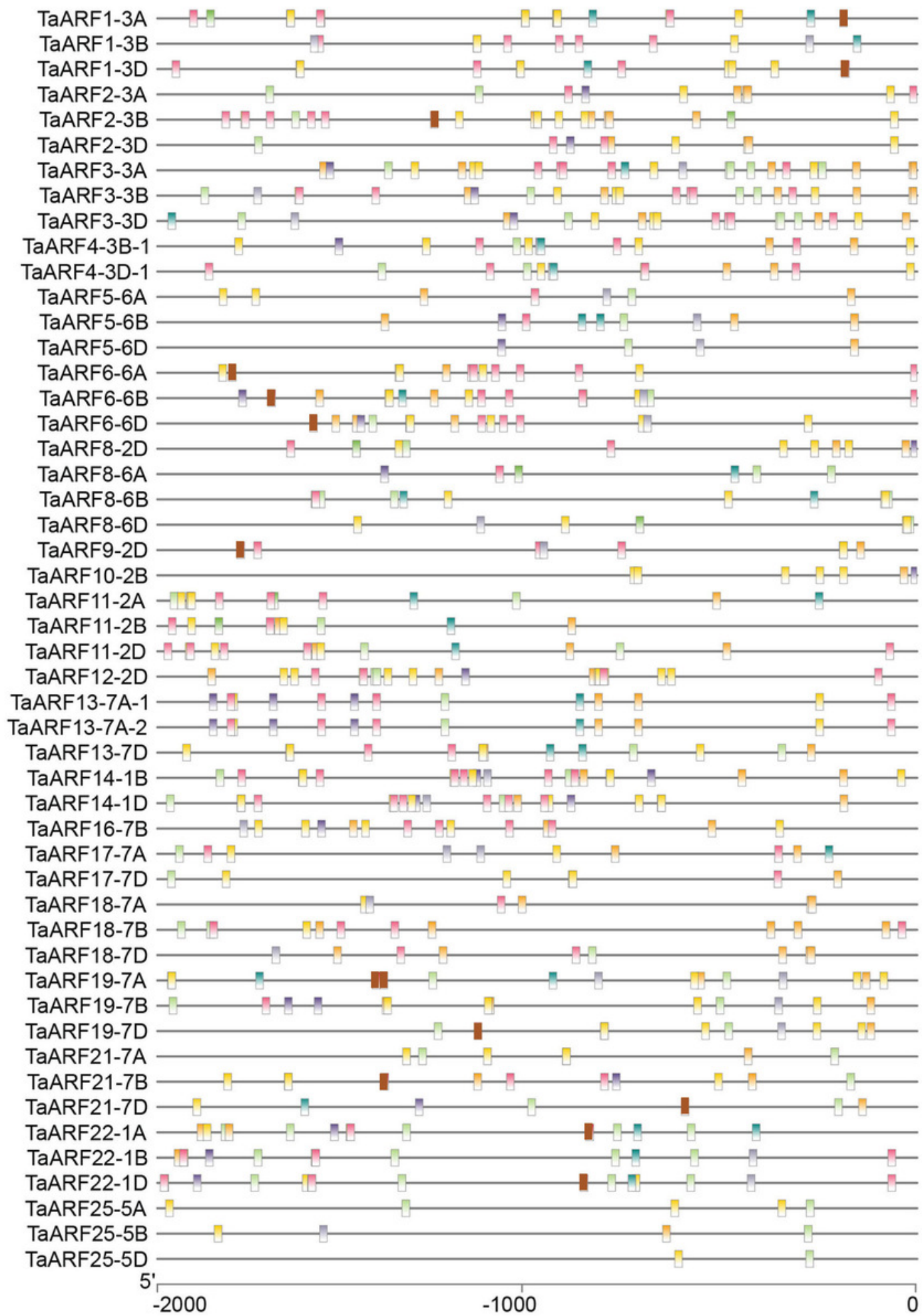

AuxRR-core ABRE CGTCA-motif P-box ERE TCA-element CAT-box CCGTCC-box TGA-element 


\section{Figure 7}

Schematic diagram of the chromosome distribution and interchromosome relationships of TaARFs.

The grey lines indicate all duplicated gene pairs in wheat, the highlighted red lines indicate probably duplicated TaARF gene pairs. 


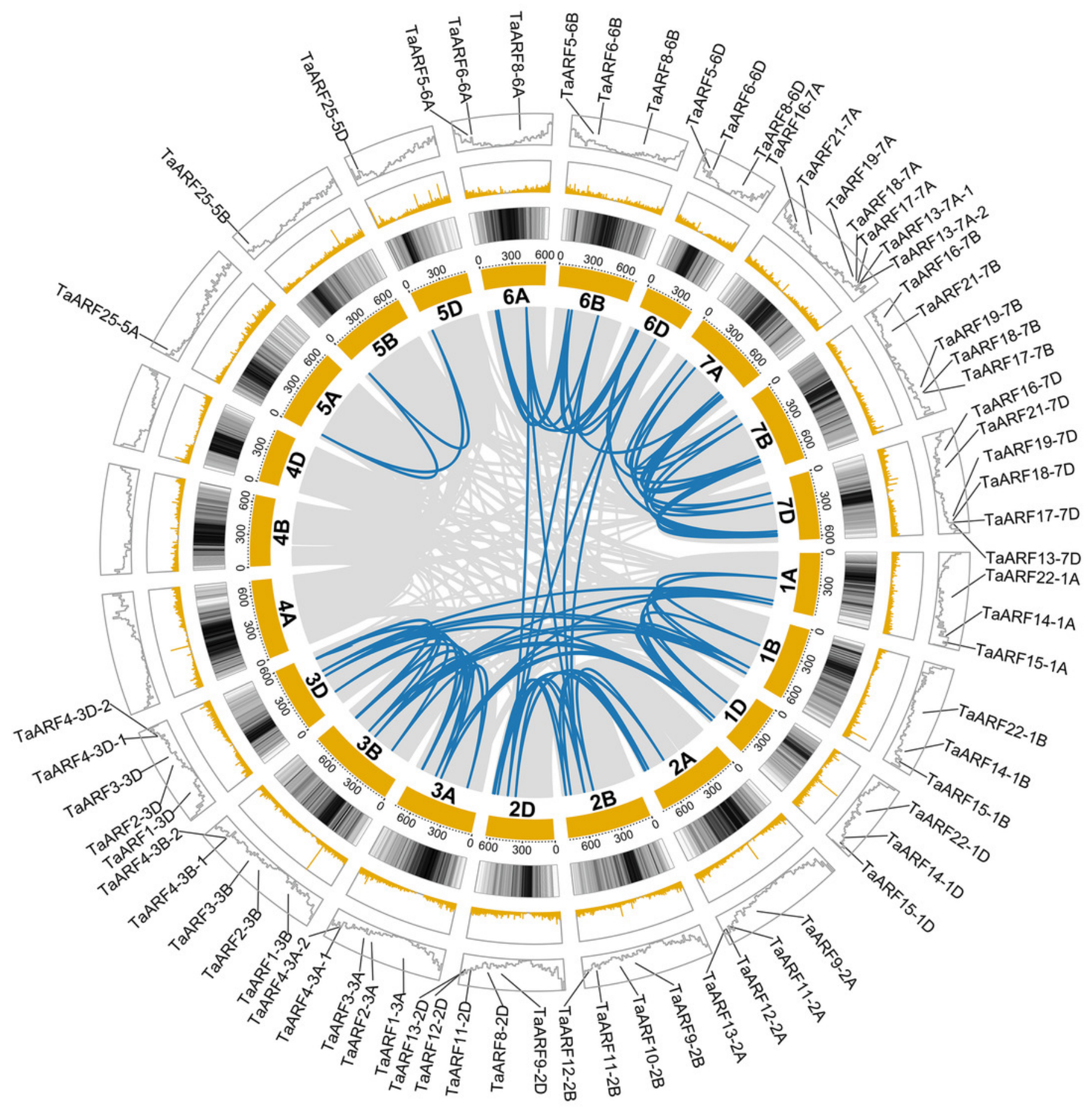


Figure 8

Syntenic relationships of $A R F$ genes between wheat and three representative species.

Gray lines in the background indicate the collinear blocks within wheat and other plant genomes, while the blue lines highlight the syntenic ARF gene pairs.
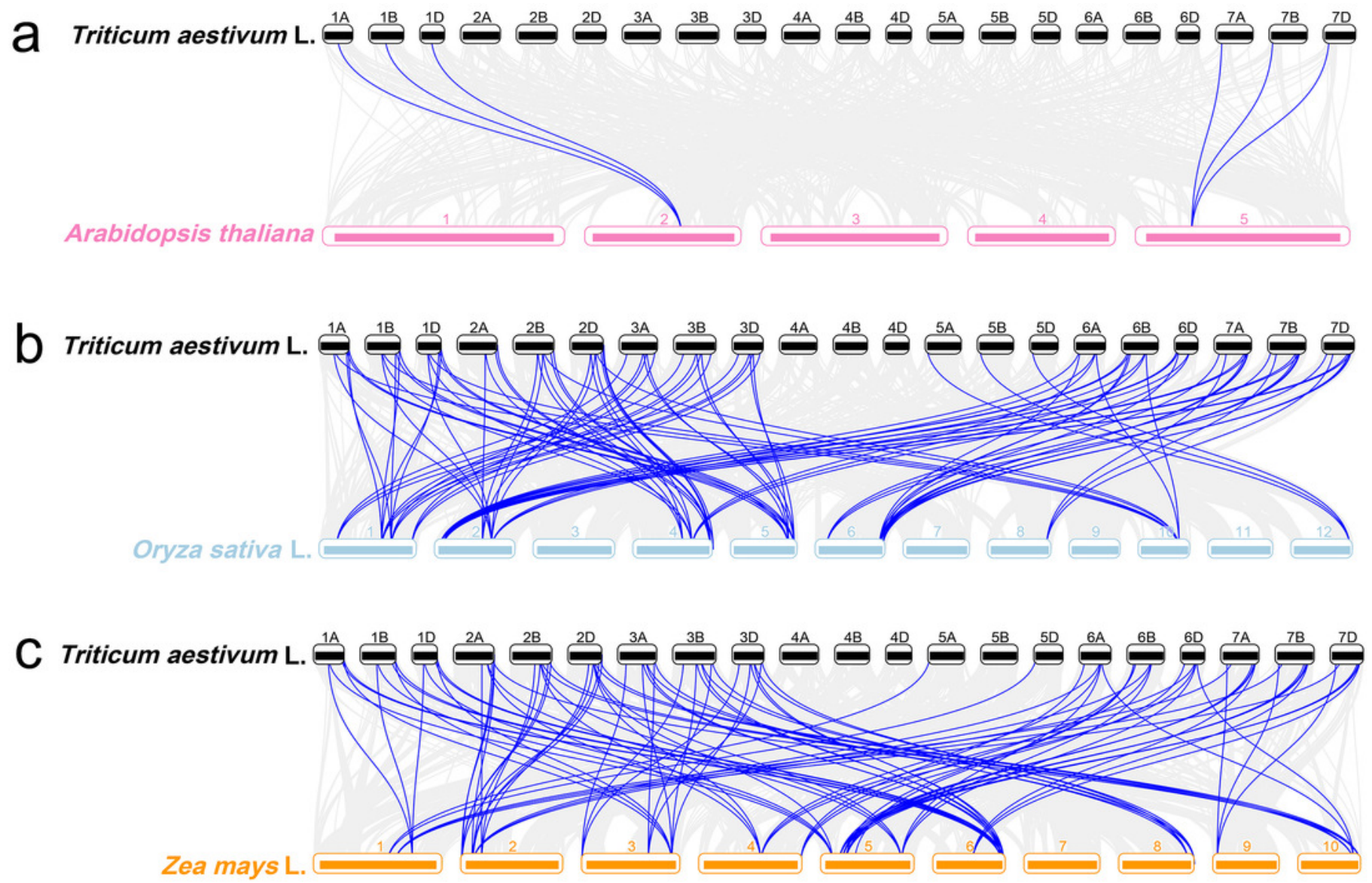
Figure 9

Expression profiles of TaARFs in various organs or tissues.

(a), Heatmap of expression profiles of TaARFs in various organs or tissues of Chinese Spring from the Wheat Expression Browser ( http://www.wheat-expression.com/ ). (b), The heat map of expression profiles of TaARFs in tiller primordia of WT and $d m c$ based on transcriptome data. Three biological replicates were set up in the mutant $d m c$ (T01, T02 and T03) and WT (T04, T05 and T06), and each sample bulk of tiller primordia included more than 10 independent individuals.
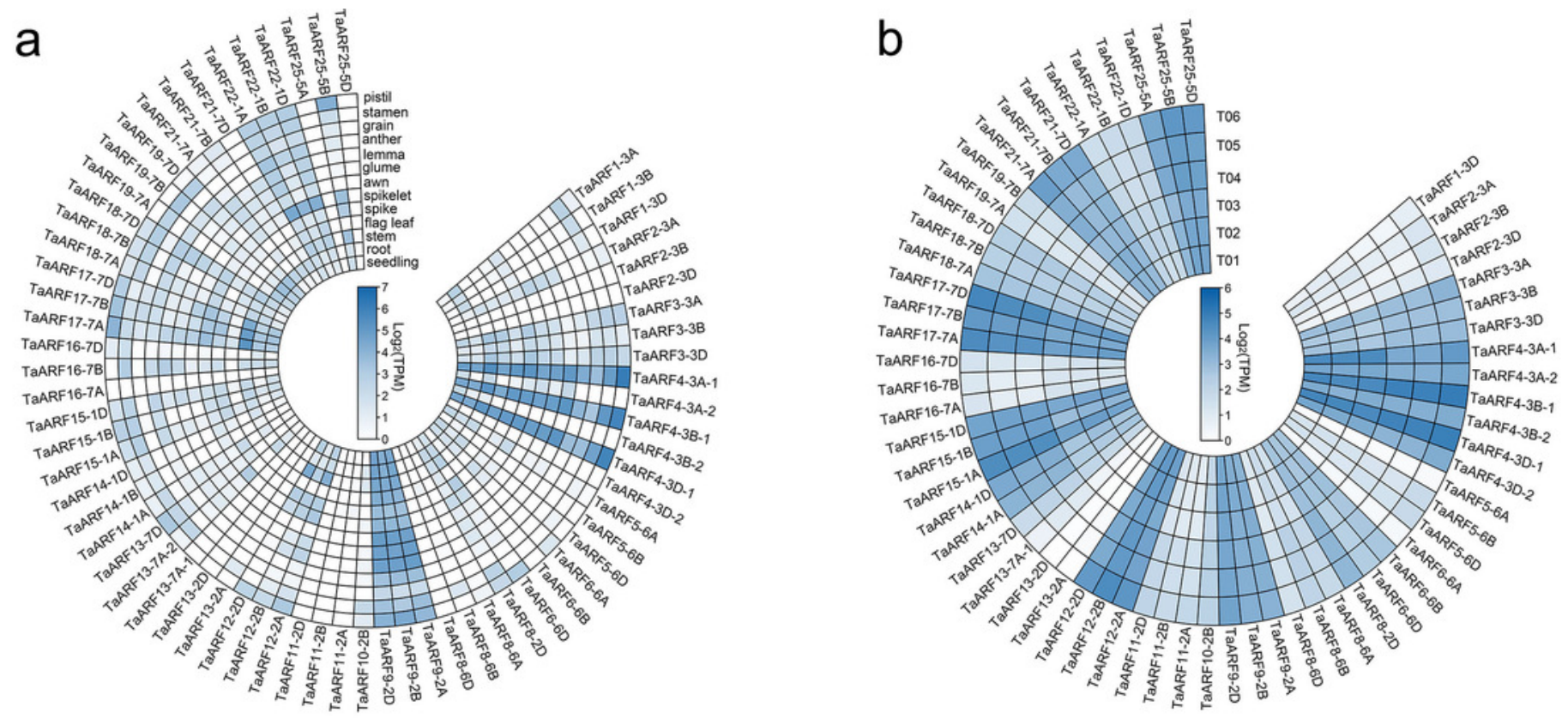
Figure 10

qRT-PCR results of 20 TaARFs in the tiller primordia of WT and $d m c$ at three tillering stages.

WT1, dmc1: the three-leaf stage; WT2, dmc2: the over-winter stage; WT3, dmc3: the rising to jointing stage. Data were normalized to $\beta$-actin gene and vertical bars indicated standard deviation. Asterisks indicate significant difference or highly significant difference between Guomai 301 and dmc.
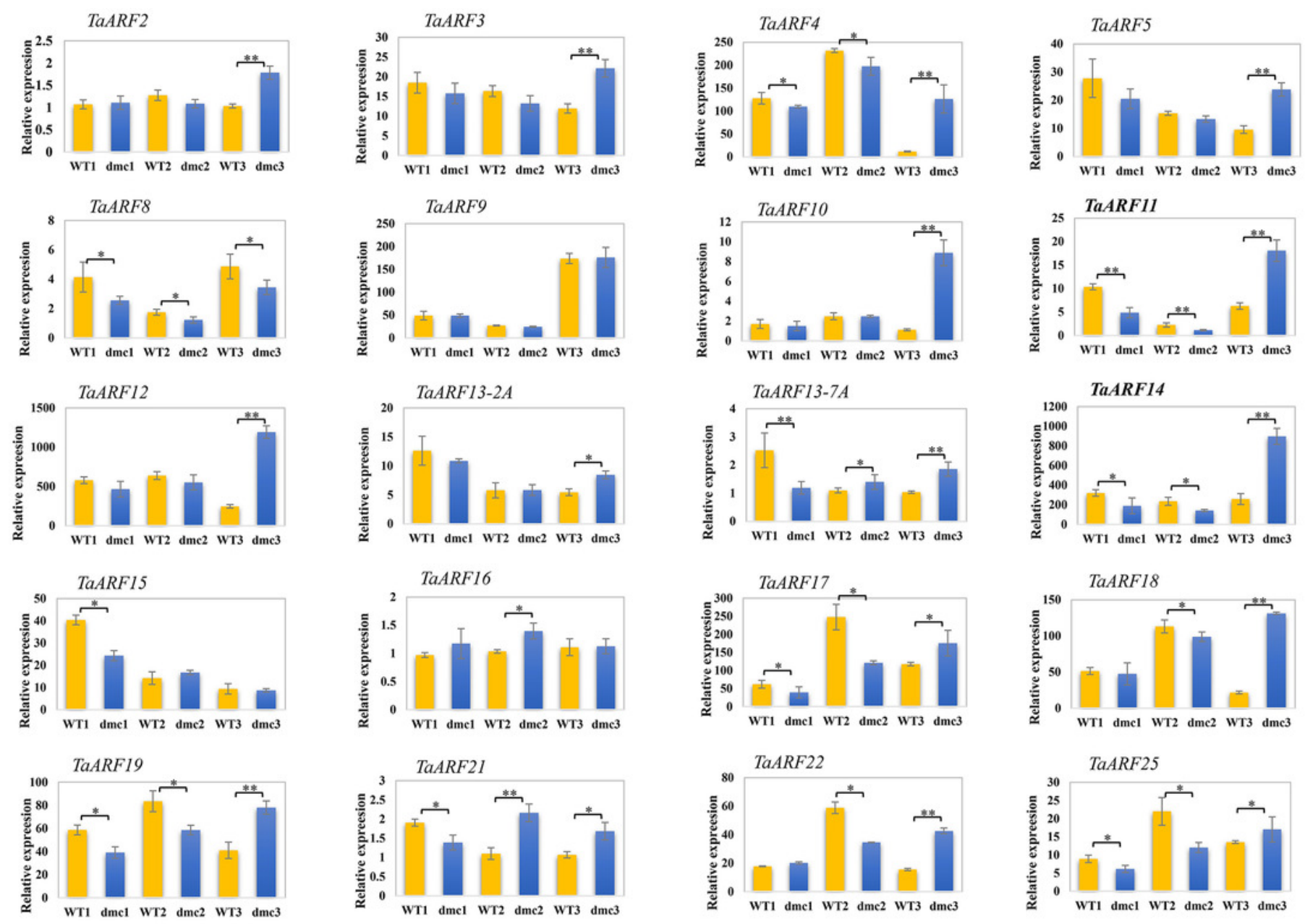

$\square \mathrm{WT} \quad \square \mathrm{dmc}$ 
Figure 11

Expression profiles of 20 TaARFs in response to IAA treatment.

Data were normalized to $\beta$-actin gene and vertical bars indicated standard deviation.

Asterisks indicate significant difference or highly significant difference between Guomai 301 and $d m c$.
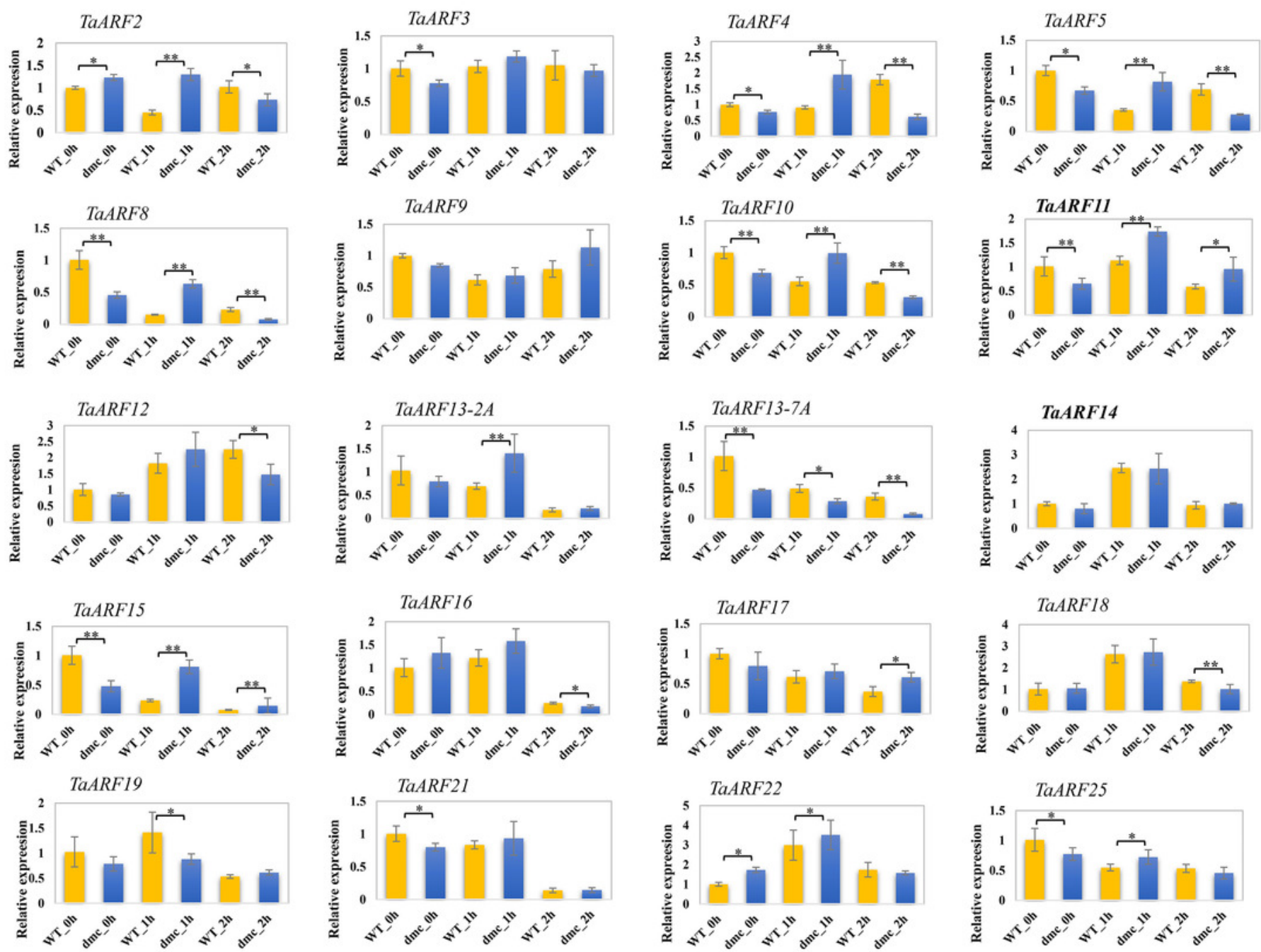

$\square \mathrm{WT} \quad \mathrm{dmc}$ 